\title{
Word norms for blind and sighted subjects: Familiarity, concreteness, meaningfulness, imageability, imagery modality, and word associations
}

\author{
NANCY H. KERR and THOMAS H. JOHNSON \\ Oglethorpe University, Atlanta, Georgia
}

\begin{abstract}
Normative values for word characteristics were obtained from a sample of 12 college-educated, totally congenitally blind subjects on the basis of their ratings of 161 nouns on scales of familiar. ity, concreteness, meaningfulness, and imageability. The dominant modality of imagery for each image-evoking word and the strongest word associate for each item also were recorded. The same data were collected for a group of sighted subjects, both to provide a comparison group for the blind subjects and to test the comparability of sighted subjects' ratings with existing norms. Ratings for sighted subjects correlated strongly with those norms, although the coefficients were slightly higher for ratings of concreteness and imageability than for ratings of familiarity and meaningfulness. Ratings of blind subjects correlated only slightly lower with existing norms for imagery and concreteness, but considerably lower for familiarity and meaningfulness.
\end{abstract}

Thanks, in large part, to the pioneering work of Allan Paivio and his associates, there has been considerable interest over the past several decades in mental imagery and the effect that word values such as concreteness, imageability, and meaningfulness might have on the imagery process. This interest has resulted in the creation of a number of lists of word norms for items rated on a variety of dimensions. Primary among these normative resources is Paivio, Yuille, and Madigan's (1968) frequently cited list of ratings for 925 nouns on the dimensions of concreteness, imagery, and meaningfulness. Rubin and Friendly (1986) have recently provided additional measures of free recall, availability, goodness, emotionality, and pronounceability for these same 925 nouns. Toglia and Battig (1978) greatly expanded the pool of available word norms with an ambitious listing of 2,854 words rated on the dimensions of concreteness, imagery, categorizability, meaningfulness, familiarity, number of attributes, and pleasantness. Additional lists of word norms have been published by Friendly, Franklin, Hoffman, and Rubin (1982) on measures of imagery, concreteness, orthographic variables, and grammatical usage for 1,080 common words and by Gilhooly and Logie (1980) on age of acquisition, imagery, concreteness, familiarity, and ambiguity for 1,944 words.

This research was supported in part by a Faculty Development Grant from Oglethorpe University to the first author. We are grateful to Wendy McKelvey for her assistance in assembling the materials, to Katie Garrigan for her help in organizing and analyzing the data, and to Laura Bradley of the Cognition Research Laboratory at GMHI for her participation in the final data analysis. Requests for reprints should be sent to N. H. Kerr, Oglethorpe University, 4484 Peachtree Road, NE, Atlanta, GA 30319.
A natural outgrowth of the interest in mental imagery has been a curiosity about the nature of the imagery process in people who have experienced the world in the absence of one of the sensory modalities, for example, blind or deaf individuals. Researchers who have studied blind subjects have been interested both in whether imagery instructions and words with high imagery value result in better memory as they do for sighted subjects and in whether imagery modality affects memorability of items differently for the two subject populations.

Since word norms have been available only for sighted subjects, researchers have developed a variety of strategies for selecting appropriate items for use with blind subjects. Some researchers (e.g., Craig, 1973) have relied directly on sighted norms such as those developed by Paivio et al. (1968), while others (e.g., Jonides, Kahn, \& Rozin, 1975) have selected items from those norms and asked their own subjects to rate the items on such characteristics as imagery modality. In an early study on the effect of imagery modality on memory for imaged items, Paivio and Okovita (1971) selected many of their items from Yuille and Barnsley's (1969) unpublished ratings of words based on ease of auditory, tactile, and visual imagery and added to them a number of items they judged to be easily imaged in either the auditory or visual modality. Subsequent researchers (e.g., Hans, 1974) have employed Paivio and Okovita's word lists, sometimes adding items based on Paivio et al.'s ratings and their own judgments of imagery modality (e.g., Zimler \& Keenan, 1983). Marchant and Malloy (1984) selected items from Yuille and Barnsley's list and expanded on it by having additional words rated using the same procedures. Researchers concerned about the familiarity of words and 
their referents to the blind have selected items of high and low familiarity based on personal judgment and experience (Cornoldi, Calore, \& Pra-Baldi, 1979; Cornoldi, DeBeni, Roncari, \& Romano, 1989; Kerr, 1983), on judgments of blind assistants (Sholl \& Easton, 1986), or on the judgments of teachers of the blind (DeBeni \& Cornoldi, 1988).

The continued interest in mental imagery and cognitive processes in the blind, coupled with increasing concerns about the comparability of word meaning and familiarity for blind and sighted subjects, suggested to us the advisability of establishing a pool of words rated for normative values by both blind and sighted subjects. Because the number of totally congenitally blind subjects available for the rating task was limited, the rating procedures we employed were slightly different from those used in previous collections of normative values. All subjects rated all words on all dimensions, with blocked presentation and counterbalancing procedures to reduce the effect of one rating task on another. Normative values were collected only for the dimensions considered relevant by previous researchers interested in mental imagery and cognitive processes of blind subjects. Likewise, only words used in previous studies of the blind were included. A total of 161 nouns were rated by both blind and sighted subjects on the dimensions of familiarity, concreteness, meaningfulness, and imageability. The dominant modality of imagery and first associated word were also recorded.

\section{METHOD}

\section{Subjects}

Twelve ( 8 female, 4 male) college-educated blind adults, ranging in age from 33 to 49 years, were paid participants. All subjects were blind from birth or became blind within the first 2 years of life and reported no memory of visual experience. All were totally blind or had minimal light perception, and the causes of blindness were exclusive of cortical damage. Twelve ( 6 female, 6 male) sighted college-educated adults and current college students, ranging in age from 18 to 33 , also participated for pay.

\section{Materials}

One hundred sixty-one nouns were selected from the items used in previous research studies on imagery in the blind (Jonides et al., 1975; Paivio \& Okovita, 1971; Sholl \& Easton, 1986; Zimler \& Keenan, 1983). The nouns were arbitrarily divided into two lists, with the stipulation that related words such as daybreak and daylight appeared on different lists. Each list was recorded on tape in both alphabetical and reverse-alphabetical order to reduce potential order effects.

The instructions for rating the familiarity, concreteness, meaningfulness, and imageability of words were closely adapted from those used by Toglia and Battig (1978), with a scale of 1 to 7 used for all ratings. Following the instructions for rating the imageability of words, the sub- jects received the following instructions for reporting the imagery modality.

Once you have given your rating for imagery on the 1 to 7 scale, I would then like you to judge the primary sensory modality of the mental image you created when you heard the word. In other words, was your mental image:

(a) Visual: it was most like seeing it with your eyes

(b) Auditory: it was most like hearing it with your ears

(c) Tactile: it was most like touching it with your hands or another part of your body

(d) Taste: it was most like tasting it on your tongue

(e) Smell: it was most like smelling it with your nose

(f) Spatial: you were most aware of the physical qualities such as size, shape, and position in space, but the image was not tied to a particular sensory system

(g) or did the word arouse no mental imagery of any kind, as indicated by your rating of 1 on the 1 to 7 scale.

So, after hearing each word on tape, first give your rating for imagery on the 1 to 7 scale; and then give the primary sensory modality of your mental image, either visual, spatial, auditory, tactile, smell, or no mental imagery of any kind.

The following instructions for word association were presented along with the instructions for rating word familiarity.

I am also interested in the first related word that comes to your mind as you hear each of the words on tape. Therefore, the first thing you should do after hearing each taped word is to say the first word that comes to your mind. For example, when you hear the word "penny" you might immediately think of the word "coin." Please do not stop to reflect or select the associate word. There are no right or wrong answers, and we are interested in your most immediate association.

\section{Procedure}

Each experimental session began with the rating of word familiarity and production of word associates for all 161 nouns, since these seemed to be the two dimensions most likely to be affected by repeated exposure to the words. The experimenter read the instructions to the subject and answered any questions, then played the words on tape one at a time and recorded the subject's verbal response. The order of presentation of the subsequent three rating tasks was counterbalanced across subjects, as was the order of presentation of tapes. The subjects always heard the instructions and rated all 161 nouns on the relevant dimension before proceeding to the next task. The entire rating session lasted from 2-3 h. 
Table 1

Number of Words for Which Blind or Sighted Subjects Gave the Higher Rating

\begin{tabular}{lcccc}
\hline \multirow{2}{*}{$\begin{array}{c}\text { Group with } \\
\text { Higher Rating }\end{array}$} & \multicolumn{4}{c}{ Rating Category } \\
\cline { 2 - 5 } & Familiar & Concrete & Meaningful & Image \\
\hline Blind & 155 & 87 & 68 & 80 \\
Tie & 2 & 4 & 7 & 0 \\
Sighted & 4 & 70 & 86 & 81 \\
\hline
\end{tabular}

\section{RESULTS}

A summary of the ratings, word associates, and imagery modalities is presented in the Appendix. Correlation coefficients for the mean ratings of blind and sighted subjects on the 161 words were relatively high for the dimensions of concreteness (.86) and imageability (.84), but noticeably lower for familiarity (.56) and meaningfulness (.51). For the 80 items that appeared both on our list and on Toglia and Battig's (1978) list, correlation coefficients for familiarity and meaningfulness were .76 and .65 for our sighted subjects and .48 and .36 for our blind subjects. On the dimensions of concreteness and imageability, our subjects' mean ratings were correlated with those of Toglia and Battig, Paivio et al. (1968; 119 shared items), and Friendly et al. (1982; 38 shared items). For sighted subjects, the correlations were $.92, .95$, and .82 for concreteness and $.80, .95$, and .90 for imageability. For blind subjects, the correlations were $.75, .93$, and .61 for concreteness and $.61, .86$, and .66 for imageability.

The blind subjects had a strong tendency to rate the familiarity of the words with a higher scale value than sighted subjects. For only 4 of the 161 nouns did sighted subjects give a higher familiarity rating than did blind subjects (mast, mirror, painting, rainbow), and ratings were tied for only 2 (sky, sunset). Relative scale values for the two subject groups were more similar for the other three rating scales (see Table 1).

\section{DISCUSSION}

Blind and sighted subjects in our study were more consistent with each other in their ratings of concreteness and imageability of words than in their ratings of familiarity and meaningfulness. The low correlations for the latter two dimensions probably reflect in part the differences in the reliability of ratings on these dimensions in general. Belleza, Greenwald, and Banaji (1986), for example, reported correlation coefficients very similar to those for our sighted subjects for familiarity (.72) and imageability $(.85)$ when they correlated their ratings with those of Toglia and Battig (1978). However, the familiarity and meaningfulness correlations for our blind subjects both with our sighted subjects and with Toglia and Battig's sighted subjects were considerably lower than the correlation between the two groups of sighted subjects. This fact suggests that visual experience contributes to these ratings and that blind and sighted subjects differ in their judgments of familiarity and meaningfulness.
The overall tendency for the blind subjects to rate items higher than the sighted subjects on the familiarity scale but not on the other three scales may result from the auditory presentation of the words. Since the auditory modality is more predominant for blind subjects than for sighted subjects, it is possible that the sounds of words appear more familiar to blind people who hear them frequently than to sighted people who frequently read them without attending to their phonetic qualities. There is nothing in the data to suggest that words that were rated especially high in familiarity by the blind subjects as compared with the sighted subjects differed systematically in their other scale values. Some words (e.g., goblet, growl) showed values on all scales that were higher for the blind subjects than for the sighted subjects. Others (e.g., animosity, devotion) were higher for the blind subjects only on the familiarity dimension, with similar values for the blind and sighted subjects on the other three scales. The most puzzling cases were relatively high for the blind subjects on familiarity but relatively low (e.g., maiden) or mixed (e.g., meadow) on the other three scales.

Differences between the blind and sighted subjects on ratings of concreteness were based in part on the higher ratings given by blind subjects to words with a strong auditory connection. Of the 24 words that blind subjects gave a rating of 1 scale point or more higher than sighted subjects, 20 had a clear auditory component (bang, beep, clang, click, conversation, echo, gong, groan, growl, hiss, laughter, pop, roar, scream, shout, snap, song, squeak, tone, whisper). Auditory characteristics apparently also affected relative meaningfulness and imageability ratings for the blind and sighted subjects; 10 of 18 words with differences on the meaningfulness scale of 1 point or more referred to auditorily experienced items (accordion, beep, clang, click, gong, growl, pop, rattle, roar, tone), and 11 of 14 words on the imageability scale (clang, conversation, gong, groan, pop, rattle, roar, shout, snap, whistle).

One potentially surprising result in the selection of imagery modality was the use of the term visual by blind people to describe their imagery experience. We had included the term spatial as a modality choice to provide blind subjects with an alternative to the visual category. Yet, despite reminders from the experimenter that visual meant "like seeing it with your eyes," several blind subjects persisted in using the term to describe the imagery modality. For these subjects, whose daily experience includes terms such as "visualizing," "looking," or "seeing" with reference to their own experiences, the term visual is a natural one, probably much more natural than spatial. This use of the term visual by totally congenitally blind subjects to describe imagery experience has been noted by other researchers as well (e.g., Marchant \& Malloy, 1984; Schlaegel, 1953). Yet, our blind subjects did not use the term visual as frequently as sighted subjects, and there appears to be a pattern in the kinds of items for which they selected the term visual. The word for which the largest number of blind subjects $(50 \%)$ listed the visual modality was headlight, which might actually 
have been experienced visually by those subjects who had had light perception at some time during their lives. The common denominator for words that received visual ratings from $42 \%$ of the blind subjects (daylight, green), $33 \%$ of the blind subjects (daybreak, galaxy, painting, poster, scarlet, shadow, stain, star), and $25 \%$ of the blind subjects (acrobat, dawn, dragon, flag, hillside, juggler, meadow, mirror, portrait, rainbow, roof, sky, sunset, valley) is that the distinctive physical characteristics of the words' referents can be experienced only through the visual sense. In contrast, there were many words that received predominantly visual ratings from the sighted subjects but for which no blind subject reported a visual image (e.g., arm, ball, blossom, cane, cave, coin, flower, potato, singer, speaker, skillet). All of these items probably had been experienced by the blind subjects through sensory modalities other than vision and were experienced imaginally through the familiar nonvisual sense modalities. Thus it appears that the term "visual image" was reserved by the blind subjects for those items whose appearance they could imagine but with which they had no direct sensory experience. These items are likely to have been described to them in visual terms, making this the sensible choice for imagery modality.

It is also interesting to note that some words were strongly associated with "visual" words for the blind subjects as well as the sighted subjects. For example, lightning was strongly associated with thunder, blue with sky, red with scarlet and with blood, green with meadow, and colors with rainbow. This may help to explain why previous researchers (e.g., Zimler \& Keenan, 1983) have found that color names serve as effective clustering categories for both blind and sighted subjects.

Despite the high correlations of the ratings of our subjects with those from previous normative samples, a word of caution is in order regarding both the generalizability and the variability of the data presented here. The norms were collected from a select subgroup of blind people who are college educated and totally congenitally blind. These criteria were selected as representing both maximal homogeneity in the critical area of visual experience and the population most frequently studied by researchers interested in the cognitive consequences of blindness. Limited populations, however, also mean limited availability of samples from those populations, and the sample size here is therefore small. Although there is little reason to believe that collecting additional data from comparable subjects would increase measures of variability (standard deviations), it is important to note that confidence intervals around our means are relatively large. Thus, particularly for items of intermediate scale value, researchers may wish to supplement the ratings given here with additional ratings given by their own subjects after they have participated in an experiment. However, this caution is less applicable to those items most likely to be useful in subsequent research, namely items of relatively low or relatively high scale values.

\section{REFERENCES}

Belleza, F. S., Greenwald, A. G., \& Banain, M. R. (1986). Words high and low in pleasantness as rated by male and female college students. Behavior Research Methods, Instruments, \& Computers, 18 , 299-303.

Cornoldi, C., Calore, D., \& Pra-Baldi, A. (1979). Imagery ratings and recall in congenitally blind subjects. Perceptual \& Motor Skills, 48, 627-639.

Cornoldi, C., DeBeni, R., Roncari, S., \& Romano, S. (1989). The effects of imagery instructions on total congenital blind recall. European Journal of Cognitive Psychology, 1, 321-331.

CraIG, E. (1973). Role of mental imagery in free recall of deaf, blind, and normal subjects. Journal of Experimental Psychology, 97, 249-253.

DEBENI, R., \& CORNOLDI, C. (1988). Imagery limitations in totally congenitally blind subjects. Journal of Experimental Psychology: Learning, Memory, \& Cognition, 14, 650-655.

Friendly, M., Franklin, P. E., Hoffman, D., \& Rubin, D. C. (1982). The Toronto word pool: Norms for imagery, concreteness, orthographic variables, and grammatical usage for 1,080 words. Behavior Research Methods \& Instrumentation, 14, 375-399.

GilHooly, K. J., \& LOGIE, R. H. (1980). Age-of-acquisition, imagery, concreteness, familiarity, and ambiguity measures for 1,944 words. Behavior Research Methods \& Instrumentation, 12, 395-427.

HaNs, M. A. (1974). Imagery and modality in paired-associate learning in the blind. Bulletin of the Psychonomic Society, 4, 22-24.

JonIDEs, J., KAHN, R., \& RozIN, P. (1975). Imagery instructions improve memory in blind subjects. Bulletin of the Psychonomic Society, 5, 424-426.

KERR, N. H. (1983). The role of vision in "visual imagery" experiments: Evidence from the congenitally blind. Journal of Experimental Psychology: General, 112, 265-277.

MARChANT, B., \& MaLLoY, T. E. (1984). Auditory, tactile, and visual imagery in PA learning by congenitally blind, deaf, and normal adults. Journal of Mental Imagery, 8, 19-32.

Parvio, A., \& OxovitA, H. W. (1971). Word imagery modalities and associative learning in blind and sighted subjects. Journal of Verbal Learning \& Verbal Behavior, 10, 506-510.

Paivio, A., Yullee, J. C., \& Madigan, S. A. (1968). Concreteness, imagery, and meaningfulness values for 925 nouns. Journal of Experimental Psychology Monographs, 76(1, Pt. 2).

RuBIN, D. C., \& FRIENDLY, M. (1986). Predicting which words get recalled: Measures of free recall, availability, goodness, emotionality, and pronunciability for 925 nouns. Memory \& Cognition, 14, 79-94.

SCHLAEGEL, T. F., JR. (1953). The dominant method of imagery in blind as compared to sighted adolescents. Journal of Genetic Psychology, 83, 265-277.

Sholl, M. J., \& Easton, R. D. (1986). Effect of object familiarity on verbal learning in the sighted and the blind. Joumal of Experimental Psychology: Learning, Memory, \& Cognition, 12, 190-200.

TogliA, M. P., \& BATTIG, W. F. (1978). Handbook of semantic word norms. Hillsdale, NJ: Erlbaum.

Yuille, J. C., \& BaRNSLEY, R. H. (1969). [Norms for 590 nouns.] Unpublished raw data. (Available from J. C. Yuille, University of British Columbia, Vancouver, British Columbia, Canada)

Zimler, J., \& KeENAN, J. M. (1983). Imagery in the congenitally blind: How visual are visual images? Journal of Experimental Psychology: Learning, Memory, \& Cognition, 9, 269-282. 
APPENDIX

The 161 nouns are listed in alphabetical order, followed by the mean score and below it by the standard deviation for the ratings of familiarity (f), concreteness (c), meaningfulness (m), and imagery (i). The percentage of subjects selecting each modality for imagery (visual, auditory, tactile, taste, spatial, or smell) and the two most frequent associates are presented following the ratings. Additional associates are included in the case of ties, and no associate is listed if there was lack of agreement between at least two subjects.

\begin{tabular}{|c|c|c|c|c|c|c|c|c|c|c|c|c|c|}
\hline \multirow[b]{2}{*}{ Noun } & \multirow{2}{*}{$\begin{array}{c}\text { Subject } \\
\text { Group }\end{array}$} & \multirow[b]{2}{*}{$\mathrm{f}$} & \multirow[b]{2}{*}{ c } & \multirow[b]{2}{*}{$\mathrm{m}$} & \multirow[b]{2}{*}{ i } & \multicolumn{6}{|c|}{ Modality (\%) } & \multirow{2}{*}{$\begin{array}{c}\text { Strongest } \\
\text { Associates }\end{array}$} & \multirow[b]{2}{*}{$\%$} \\
\hline & & & & & & (v & a t & tc & ts & sp & sm) & & \\
\hline \multirow[t]{2}{*}{ Accordion } & B & $\begin{array}{l}6.25 \\
1.14\end{array}$ & $\begin{array}{l}6.25 \\
1.22\end{array}$ & $\begin{array}{l}5.00 \\
1.71\end{array}$ & $\begin{array}{l}6.50 \\
0.80\end{array}$ & - & 831 & 17 & - & - & - & $\begin{array}{l}\text { Instrument } \\
\text { Music }\end{array}$ & $\begin{array}{l}42 \\
33\end{array}$ \\
\hline & $\mathrm{s}$ & $\begin{array}{l}4.75 \\
1.76\end{array}$ & $\begin{array}{l}6.08 \\
0.79\end{array}$ & $\begin{array}{l}3.75 \\
1.48\end{array}$ & $\begin{array}{l}5.58 \\
1.24\end{array}$ & 50 & 50 & - & - & - & - & $\begin{array}{l}\text { Instrument } \\
\text { Monkey }\end{array}$ & $\begin{array}{l}25 \\
17\end{array}$ \\
\hline \multirow[t]{2}{*}{ Acrobat } & B & $\begin{array}{l}6.08 \\
1.16\end{array}$ & $\begin{array}{l}4.58 \\
2.23\end{array}$ & $\begin{array}{l}3.42 \\
1.73\end{array}$ & $\begin{array}{l}4.00 \\
1.86\end{array}$ & 25 & 17 & 17 & - & 25 & - & $\begin{array}{l}\text { Circus } \\
\text { Gymnast }\end{array}$ & $\begin{array}{l}33 \\
25\end{array}$ \\
\hline & S & $\begin{array}{l}5.00 \\
1.60\end{array}$ & $\begin{array}{l}5.50 \\
1.45\end{array}$ & $\begin{array}{l}4.25 \\
1.06\end{array}$ & $\begin{array}{l}5.58 \\
1.24\end{array}$ & 100 & - & - & - & - & - & $\begin{array}{l}\text { Circus } \\
\text { Gymnast }\end{array}$ & $\begin{array}{l}33 \\
25\end{array}$ \\
\hline \multirow[t]{2}{*}{ Affection } & B & $\begin{array}{l}6.75 \\
0.45\end{array}$ & $\begin{array}{l}3.83 \\
2.44\end{array}$ & $\begin{array}{l}5.17 \\
1.95\end{array}$ & $\begin{array}{l}4.42 \\
2.47\end{array}$ & 08 & - & 67 & - & - & - & $\begin{array}{l}\text { Love } \\
\text { Feel ing }\end{array}$ & $\begin{array}{l}50 \\
17\end{array}$ \\
\hline & $\mathbf{S}$ & $\begin{array}{l}5.58 \\
1.73\end{array}$ & $\begin{array}{l}3.92 \\
2.23\end{array}$ & $\begin{array}{l}5.00 \\
1.65\end{array}$ & $\begin{array}{l}3.42 \\
2.23\end{array}$ & 17 & -4 & 42 & - & 17 & - & $\begin{array}{l}\text { Love } \\
\text { Care }\end{array}$ & $\begin{array}{l}42 \\
25\end{array}$ \\
\hline \multirow[t]{2}{*}{ Amplifier } & B & $\begin{array}{l}5.92 \\
1.16\end{array}$ & $\begin{array}{l}6.00 \\
1.54\end{array}$ & $\begin{array}{l}4.33 \\
1.78\end{array}$ & $\begin{array}{l}5.33 \\
2.23\end{array}$ & - & 750 & 08 & - & - & - & $\begin{array}{l}\text { Loud } \\
\text { Speaker }\end{array}$ & $\begin{array}{l}25 \\
25\end{array}$ \\
\hline & $\mathrm{s}$ & $\begin{array}{l}5.00 \\
1.65\end{array}$ & $\begin{array}{l}5.58 \\
1.56\end{array}$ & $\begin{array}{l}3.67 \\
1.23\end{array}$ & $\begin{array}{l}4.75 \\
2.01\end{array}$ & 33 & 50 & - & - & - & - & $\begin{array}{l}\text { Music } \\
\text { Speaker }\end{array}$ & $\begin{array}{l}25 \\
17\end{array}$ \\
\hline \multirow[t]{2}{*}{ Animosity } & B & $\begin{array}{l}5.92 \\
1.38\end{array}$ & $\begin{array}{l}2.83 \\
2.12\end{array}$ & $\begin{array}{l}4.00 \\
1.86\end{array}$ & $\begin{array}{l}2.33 \\
2.15\end{array}$ & - & 17 & 08 & - & - & - & $\begin{array}{l}\text { Anger } \\
\text { Hate }\end{array}$ & $\begin{array}{l}25 \\
25\end{array}$ \\
\hline & S & $\begin{array}{l}4.08 \\
1.73\end{array}$ & $\begin{array}{l}2.75 \\
1.71\end{array}$ & $\begin{array}{l}3.50 \\
2.15\end{array}$ & $\begin{array}{l}2.58 \\
1.93\end{array}$ & 17 & 08 & 17 & - & 08 & - & $\begin{array}{l}\text { Anger } \\
\text { Hate }\end{array}$ & $\begin{array}{l}25 \\
25\end{array}$ \\
\hline \multirow[t]{2}{*}{ Anxiety } & B & $\begin{array}{l}6.50 \\
0.67\end{array}$ & $\begin{array}{l}3.75 \\
2.26\end{array}$ & $\begin{array}{l}4.25 \\
1.91\end{array}$ & $\begin{array}{l}2.50 \\
2.35\end{array}$ & - & 17 & - & - & 17 & - & $\begin{array}{l}\text { Anxious } \\
\text { Fear }\end{array}$ & $\begin{array}{l}25 \\
17\end{array}$ \\
\hline & s & $\begin{array}{l}5.83 \\
1.11\end{array}$ & $\begin{array}{l}3.17 \\
1.80\end{array}$ & $\begin{array}{l}4.42 \\
1.93\end{array}$ & $\begin{array}{l}3.00 \\
2.30\end{array}$ & 25 & - & 17 & - & 08 & - & $\begin{array}{l}\text { Attack } \\
\text { Stress }\end{array}$ & $\begin{array}{l}25 \\
25\end{array}$ \\
\hline \multirow[t]{2}{*}{ Applause } & B & $\begin{array}{l}6.42 \\
0.90\end{array}$ & $\begin{array}{l}5.92 \\
1.00\end{array}$ & $\begin{array}{l}5.50 \\
1.24\end{array}$ & $\begin{array}{l}6.33 \\
1.07\end{array}$ & - & 100 & - & - & - & - & Clap & 92 \\
\hline & s & $\begin{array}{l}5.25 \\
1.54\end{array}$ & $\begin{array}{l}5.17 \\
1.53\end{array}$ & $\begin{array}{l}5.00 \\
1.28\end{array}$ & $\begin{array}{l}5.83 \\
1.11\end{array}$ & 17 & 83 & - & - & - & - & $\begin{array}{l}\text { Clap } \\
\text { Laughter }\end{array}$ & $\begin{array}{l}42 \\
17\end{array}$ \\
\hline
\end{tabular}


APPENDIX (Continued)

\begin{tabular}{|c|c|c|c|c|c|c|c|c|c|c|c|c|c|}
\hline \multirow[b]{2}{*}{ Noun } & \multirow{2}{*}{$\begin{array}{l}\text { Subject } \\
\text { Group }\end{array}$} & \multirow[b]{2}{*}{$\mathrm{f}$} & \multirow[b]{2}{*}{ c } & \multirow[b]{2}{*}{$\mathrm{m}$} & \multirow[b]{2}{*}{$\mathrm{i}$} & \multicolumn{6}{|c|}{ Modality (\%) } & \multirow{2}{*}{$\begin{array}{c}\text { Strongest } \\
\text { Associates }\end{array}$} & \multirow[b]{2}{*}{$\%$} \\
\hline & & & & & & $(\mathrm{v}$ & a $t$ & tc & ts & $\mathrm{sp}$ & sm) & & \\
\hline Aptitude & B & $\begin{array}{l}6.17 \\
1.27\end{array}$ & $\begin{array}{l}2.33 \\
1.78\end{array}$ & $\begin{array}{l}3.58 \\
1.56\end{array}$ & $\begin{array}{l}2.17 \\
2.12\end{array}$ & - & 08 & - & - & 08 & - & $\begin{array}{l}\text { Test } \\
\text { Ability }\end{array}$ & $\begin{array}{l}33 \\
17\end{array}$ \\
\hline & $\mathrm{s}$ & $\begin{array}{l}5.00 \\
1.48\end{array}$ & $\begin{array}{l}2.17 \\
0.83\end{array}$ & $\begin{array}{l}3.83 \\
1.34\end{array}$ & $\begin{array}{l}1.42 \\
0.79\end{array}$ & 25 & - & - & - & - & - & $\begin{array}{l}\text { Ability } \\
\text { Test }\end{array}$ & $\begin{array}{l}25 \\
17\end{array}$ \\
\hline \multirow[t]{2}{*}{ A rm } & B & $\begin{array}{l}6.92 \\
0.29\end{array}$ & $\begin{array}{l}6.67 \\
0.49\end{array}$ & $\begin{array}{l}5.75 \\
1.66\end{array}$ & $\begin{array}{l}6.08 \\
1.73\end{array}$ & - & - & 92 & - & - & - & $\begin{array}{l}\text { Leg } \\
\text { Body } \\
\text { Hand }\end{array}$ & $\begin{array}{l}25 \\
17 \\
17\end{array}$ \\
\hline & S & $\begin{array}{l}6.33 \\
0.89\end{array}$ & $\begin{array}{l}6.58 \\
0.67\end{array}$ & $\begin{array}{l}4.67 \\
1.61\end{array}$ & $\begin{array}{l}6.42 \\
0.51\end{array}$ & 92 & - & 08 & - & - & - & Leg & 50 \\
\hline \multirow[t]{2}{*}{ Atrocity } & B & $\begin{array}{l}4.92 \\
1.68\end{array}$ & $\begin{array}{l}2.25 \\
1.71\end{array}$ & $\begin{array}{l}2.67 \\
1.67\end{array}$ & $\begin{array}{l}1.42 \\
1.16\end{array}$ & - & 08 & - & - & - & - & $\begin{array}{l}\text { Bad } \\
\text { Terrible }\end{array}$ & $\begin{array}{l}17 \\
17\end{array}$ \\
\hline & $\mathrm{s}$ & $\begin{array}{l}4.42 \\
1.38\end{array}$ & $\begin{array}{l}2.42 \\
1.08\end{array}$ & $\begin{array}{l}4.42 \\
1.98\end{array}$ & $\begin{array}{l}3.17 \\
2.44\end{array}$ & 50 & $08-$ & - & - & - & - & Death & 17 \\
\hline \multirow[t]{2}{*}{ Avalanche } & B & $\begin{array}{l}5.67 \\
1.50\end{array}$ & $\begin{array}{l}4.67 \\
1.78\end{array}$ & $\begin{array}{l}3.25 \\
1.60\end{array}$ & $\begin{array}{l}4.58 \\
1.68\end{array}$ & 17 & 42 & 17 & - & 25 & - & $\begin{array}{l}\text { Snow } \\
\text { S1ide }\end{array}$ & $\begin{array}{l}25 \\
17\end{array}$ \\
\hline & S & $\begin{array}{l}4.92 \\
1.68\end{array}$ & $\begin{array}{l}4.92 \\
1.44\end{array}$ & $\begin{array}{l}4.58 \\
1.93\end{array}$ & $\begin{array}{l}5.75 \\
1.71\end{array}$ & 67 & 17 & - & - & 08 & - & $\begin{array}{l}\text { Snow } \\
\text { Rocks }\end{array}$ & $\begin{array}{l}33 \\
25\end{array}$ \\
\hline \multirow[t]{2}{*}{ Bagpipe } & B & $\begin{array}{l}5.83 \\
1.53\end{array}$ & $\begin{array}{l}6.50 \\
0.80\end{array}$ & $\begin{array}{l}4.42 \\
1.88\end{array}$ & $\begin{array}{l}6.42 \\
1.24\end{array}$ & - & 100 & - & - & - & - & $\begin{array}{l}\text { Musical } \\
\text { Instrument } \\
\text { Scottish }\end{array}$ & $\begin{array}{r}33 \\
33\end{array}$ \\
\hline & $S$ & $\begin{array}{l}5.08 \\
1.56\end{array}$ & $\begin{array}{l}5.92 \\
1.08\end{array}$ & $\begin{array}{l}3.75 \\
2.09\end{array}$ & $\begin{array}{l}5.67 \\
1.23\end{array}$ & 42 & $58-$ & - & - & - & - & $\begin{array}{l}\text { Scottish } \\
\text { Music }\end{array}$ & $\begin{array}{l}33 \\
17\end{array}$ \\
\hline \multirow[t]{2}{*}{ Ball } & B & $\begin{array}{l}6.58 \\
1.00\end{array}$ & $\begin{array}{l}6.33 \\
0.98\end{array}$ & $\begin{array}{l}5.33 \\
1.87\end{array}$ & $\begin{array}{l}6.33 \\
0.89\end{array}$ & - & 087 & 75 & - & 17 & - & Game & 17 \\
\hline & $\mathrm{s}$ & $\begin{array}{l}6.17 \\
0.83\end{array}$ & $\begin{array}{l}6.00 \\
0.95\end{array}$ & $\begin{array}{l}5.58 \\
1.68\end{array}$ & $\begin{array}{l}6.42 \\
0.79\end{array}$ & 75 & 08 & 08 & - & 08 & - & $\begin{array}{l}\text { Chain } \\
\text { Play } \\
\text { Round } \\
\text { Toy }\end{array}$ & $\begin{array}{l}17 \\
17 \\
17 \\
17\end{array}$ \\
\hline \multirow[t]{2}{*}{ Bang } & B & $\begin{array}{l}6.75 \\
0.87\end{array}$ & $\begin{array}{l}6.33 \\
0.89\end{array}$ & $\begin{array}{l}5.42 \\
1.38\end{array}$ & $\begin{array}{l}6.42 \\
0.79\end{array}$ & - & 100 & - & - & - & - & $\begin{array}{l}\text { Gun } \\
\text { Loud noise }\end{array}$ & $\begin{array}{l}50 \\
42\end{array}$ \\
\hline & $\mathrm{s}$ & $\begin{array}{l}5.33 \\
1.87\end{array}$ & $\begin{array}{l}4.58 \\
1.78\end{array}$ & $\begin{array}{l}5.25 \\
1.06\end{array}$ & $\begin{array}{l}6.00 \\
0.74\end{array}$ & 08 & 92 & - & - & - & - & Gun & 58 \\
\hline Beep & B & $\begin{array}{l}6.58 \\
0.51\end{array}$ & $\begin{array}{l}6.58 \\
0.79\end{array}$ & $\begin{array}{l}5.58 \\
1.00\end{array}$ & $\begin{array}{l}6.50 \\
1.00\end{array}$ & - & 100 & - & - & - & - & $\begin{array}{l}\text { Horn } \\
\text { Noise } \\
\text { Sound }\end{array}$ & $\begin{array}{l}33 \\
17 \\
17\end{array}$ \\
\hline
\end{tabular}


APPENDIX (Continued)

\begin{tabular}{|c|c|c|c|c|c|c|c|c|c|c|c|c|c|}
\hline \multirow[b]{2}{*}{ Noun } & \multirow{2}{*}{$\begin{array}{l}\text { Subject } \\
\text { Group }\end{array}$} & \multirow[b]{2}{*}{ f } & \multirow[b]{2}{*}{ c } & \multirow[b]{2}{*}{$\mathrm{m}$} & \multirow[b]{2}{*}{$\mathrm{i}$} & \multicolumn{6}{|c|}{ Modality (\%) } & \multirow{2}{*}{$\begin{array}{l}\text { Strongest } \\
\text { Associates }\end{array}$} & \multirow[b]{2}{*}{$\%$} \\
\hline & & & & & & $(\mathrm{v}$ & $\mathrm{a}$ & tc & ts & sp & $\mathrm{sm})$ & & \\
\hline & $\mathrm{S}$ & $\begin{array}{l}5.75 \\
1.22\end{array}$ & $\begin{array}{l}4.75 \\
1.60\end{array}$ & $\begin{array}{l}4.42 \\
1.78\end{array}$ & $\begin{array}{l}5.58 \\
1.38\end{array}$ & -1 & 100 & - & - & - & - & $\begin{array}{l}\text { Car } \\
\text { Honk }\end{array}$ & $\begin{array}{l}17 \\
17\end{array}$ \\
\hline \multirow[t]{2}{*}{ Betrayal } & B & $\begin{array}{l}6.33 \\
0.89\end{array}$ & $\begin{array}{l}2.42 \\
1.62\end{array}$ & $\begin{array}{l}3.67 \\
1.44\end{array}$ & $\begin{array}{l}2.25 \\
1.96\end{array}$ & -1 & 17 & 08 & - & 08 & - & Deception & 17 \\
\hline & $\mathrm{S}$ & $\begin{array}{l}5.00 \\
1.81\end{array}$ & $\begin{array}{l}2.42 \\
1.16\end{array}$ & $\begin{array}{l}4.75 \\
1.76\end{array}$ & $\begin{array}{l}2.00 \\
1.95\end{array}$ & 08 & - & 08 & - & 08 & - & Lie & 17 \\
\hline \multirow[t]{2}{*}{ Blessing } & $\mathrm{B}$ & $\begin{array}{l}6.58 \\
0.51\end{array}$ & $\begin{array}{l}2.92 \\
2.15\end{array}$ & $\begin{array}{l}3.92 \\
1.98\end{array}$ & $\begin{array}{l}2.83 \\
2.44\end{array}$ & -1 & 17 & - & - & 17 & - & Joy ful & 17 \\
\hline & $\mathrm{S}$ & $\begin{array}{l}5.33 \\
1.50\end{array}$ & $\begin{array}{l}3.00 \\
1.65\end{array}$ & $\begin{array}{l}3.83 \\
1.47\end{array}$ & $\begin{array}{l}2.92 \\
2.15\end{array}$ & 252 & 25 & - & 08 & - & - & Prayer & 33 \\
\hline \multirow[t]{2}{*}{ Blood } & B & $\begin{array}{l}6.58 \\
1.00\end{array}$ & $\begin{array}{l}6.08 \\
1.08\end{array}$ & $\begin{array}{l}4.92 \\
1.78\end{array}$ & $\begin{array}{l}6.17 \\
0.94\end{array}$ & 17 & - & 67 & 08 & - & 08 & Red & 58 \\
\hline & $S$ & $\begin{array}{l}5.92 \\
1.51\end{array}$ & $\begin{array}{l}6.42 \\
0.79\end{array}$ & $\begin{array}{l}6.00 \\
1.28\end{array}$ & $\begin{array}{l}6.33 \\
0.78\end{array}$ & 92 & - & 08 & - & - & - & Red & 42 \\
\hline \multirow[t]{2}{*}{ Blos som } & $\mathrm{B}$ & $\begin{array}{l}6.42 \\
0.67\end{array}$ & $\begin{array}{l}5.92 \\
1.08\end{array}$ & $\begin{array}{l}5.17 \\
1.27\end{array}$ & $\begin{array}{l}6.33 \\
0.78\end{array}$ & - & - & 58 & - & 08 & 33 & Flower & 58 \\
\hline & $\mathrm{S}$ & $\begin{array}{l}5.58 \\
1.24\end{array}$ & $\begin{array}{l}5.00 \\
1.86\end{array}$ & $\begin{array}{l}5.00 \\
1.41\end{array}$ & $\begin{array}{l}5.50 \\
1.68\end{array}$ & 75 & - & - & - & - & 25 & Flower & 50 \\
\hline \multirow[t]{2}{*}{ Boredom } & B & $\begin{array}{l}6.50 \\
0.90\end{array}$ & $\begin{array}{l}2.58 \\
1.78\end{array}$ & $\begin{array}{l}4.08 \\
1.78\end{array}$ & $\begin{array}{l}2.42 \\
2.39\end{array}$ & -1 & 17 & - & - & 08 & - & $\begin{array}{l}\text { Monotony } \\
\text { Tired }\end{array}$ & $\begin{array}{l}17 \\
17\end{array}$ \\
\hline & $\mathrm{S}$ & $\begin{array}{l}5.75 \\
1.22\end{array}$ & $\begin{array}{l}3.75 \\
2.18\end{array}$ & $\begin{array}{l}4.50 \\
1.93\end{array}$ & $\begin{array}{l}3.00 \\
2.59\end{array}$ & 17 & - & 17 & - & 17 & - & $\begin{array}{l}\text { Nothingnes } \\
\text { School }\end{array}$ & $\begin{array}{r}17 \\
17\end{array}$ \\
\hline \multirow[t]{2}{*}{ Bridge } & $B$ & $\begin{array}{l}6.33 \\
1.15\end{array}$ & $\begin{array}{l}6.08 \\
1.16\end{array}$ & $\begin{array}{l}4.92 \\
1.38\end{array}$ & $\begin{array}{l}5.67 \\
1.37\end{array}$ & 08 & - & 50 & - & 42 & - & $\begin{array}{l}\text { Creek } \\
\text { Crossing } \\
\text { River } \\
\text { Water }\end{array}$ & $\begin{array}{l}17 \\
17 \\
17 \\
17\end{array}$ \\
\hline & S & $\begin{array}{l}6.00 \\
0.95\end{array}$ & $\begin{array}{l}6.08 \\
0.90\end{array}$ & $\begin{array}{l}4.92 \\
1.56\end{array}$ & $\begin{array}{l}6.50 \\
0.52\end{array}$ & 100 & - & - & - & - & - & $\begin{array}{l}\text { Water } \\
\text { River }\end{array}$ & $\begin{array}{l}50 \\
17\end{array}$ \\
\hline \multirow[t]{2}{*}{ Butter } & B & $\begin{array}{l}6.75 \\
0.45\end{array}$ & $\begin{array}{l}6.17 \\
1.19\end{array}$ & $\begin{array}{l}5.75 \\
1.29\end{array}$ & $\begin{array}{l}6.42 \\
0.79\end{array}$ & - & - & 33 & 67 & - & - & Bread & 33 \\
\hline & $\mathrm{S}$ & $\begin{array}{l}6.33 \\
0.65\end{array}$ & $\begin{array}{l}6.00 \\
0.95\end{array}$ & $\begin{array}{l}4.83 \\
1.75\end{array}$ & $\begin{array}{l}5.67 \\
1.44\end{array}$ & 33 & - & 08 & 58 & - & - & $\begin{array}{l}\text { Bread } \\
\text { Knife }\end{array}$ & $\begin{array}{l}25 \\
25\end{array}$ \\
\hline
\end{tabular}


APPENDIX (Continued)

\begin{tabular}{|c|c|c|c|c|c|c|c|c|c|c|c|c|c|}
\hline \multirow[b]{2}{*}{ Noun } & \multirow{2}{*}{$\begin{array}{l}\text { Subject } \\
\text { Group }\end{array}$} & \multirow[b]{2}{*}{ f } & \multirow[b]{2}{*}{ c } & \multirow[b]{2}{*}{ m } & \multirow[b]{2}{*}{$\mathrm{i}$} & \multicolumn{6}{|c|}{ Modality (\%) } & \multirow{2}{*}{$\begin{array}{c}\text { Strongest } \\
\text { Associates }\end{array}$} & \multirow[b]{2}{*}{$\%$} \\
\hline & & & & & & $(\mathrm{v}$ & a & tc & ts & $\mathbf{s p}$ & $\mathrm{sm})$ & & \\
\hline \multirow[t]{2}{*}{ Candle } & B & $\begin{array}{l}6.67 \\
0.65\end{array}$ & $\begin{array}{l}5.67 \\
1.44\end{array}$ & $\begin{array}{l}5.00 \\
1.48\end{array}$ & $\begin{array}{l}6.17 \\
0.83\end{array}$ & 17 & - & 50 & - & 17 & 17 & $\begin{array}{l}\text { Light } \\
\text { Wax }\end{array}$ & $\begin{array}{l}75 \\
17\end{array}$ \\
\hline & $\mathrm{S}$ & $\begin{array}{l}6.17 \\
0.83\end{array}$ & $\begin{array}{l}5.83 \\
1.11\end{array}$ & $\begin{array}{l}4.83 \\
1.75\end{array}$ & $\begin{array}{l}5.92 \\
1.24\end{array}$ & 83 & - & 08 & - & - & 08 & $\begin{array}{l}\text { Light } \\
\text { Elame }\end{array}$ & $\begin{array}{l}42 \\
17\end{array}$ \\
\hline \multirow[t]{2}{*}{ Cane } & $\mathrm{B}$ & $\begin{array}{l}6.75 \\
0.45\end{array}$ & $\begin{array}{l}6.25 \\
1.48\end{array}$ & $\begin{array}{l}4.50 \\
1.73\end{array}$ & $\begin{array}{l}5.75 \\
1.71\end{array}$ & - & - & 83 & - & 08 & - & $\begin{array}{l}\text { Walk } \\
\text { Stick }\end{array}$ & $\begin{array}{l}33 \\
17\end{array}$ \\
\hline & $\mathrm{S}$ & $\begin{array}{l}5.33 \\
1.30\end{array}$ & $\begin{array}{l}5.50 \\
1.09\end{array}$ & $\begin{array}{l}4.25 \\
1.48\end{array}$ & $\begin{array}{l}5.67 \\
1.15\end{array}$ & 83 & - & 08 & - & 08 & - & $\begin{array}{l}\text { Able } \\
\text { Old } \\
\text { Walk }\end{array}$ & $\begin{array}{l}17 \\
17 \\
17\end{array}$ \\
\hline \multirow[t]{2}{*}{ Cave } & B & $\begin{array}{l}6.08 \\
1.38\end{array}$ & $\begin{array}{l}5.50 \\
1.51\end{array}$ & $\begin{array}{l}3.92 \\
1.78\end{array}$ & $\begin{array}{l}5.25 \\
1.66\end{array}$ & - & 17 & 42 & - & 42 & - & $\begin{array}{l}\text { Undergroun } \\
\text { Cold } \\
\text { Dark } \\
\text { Man }\end{array}$ & $\begin{array}{r}\text { d } 25 \\
17 \\
17 \\
17\end{array}$ \\
\hline & $\mathrm{s}$ & $\begin{array}{l}5.58 \\
1.31\end{array}$ & $\begin{array}{l}5.67 \\
1.07\end{array}$ & $\begin{array}{l}5.00 \\
1.65\end{array}$ & $\begin{array}{l}5.58 \\
1.24\end{array}$ & 83 & - & - & - & 17 & - & $\begin{array}{l}\text { Bear } \\
\text { Dark } \\
\text { Man }\end{array}$ & $\begin{array}{l}33 \\
17 \\
17\end{array}$ \\
\hline \multirow[t]{3}{*}{ Chance } & $\mathrm{B}$ & $\begin{array}{l}6.67 \\
0.89\end{array}$ & $\begin{array}{l}2.17 \\
1.75\end{array}$ & $\begin{array}{l}4.00 \\
1.88\end{array}$ & $\begin{array}{l}2.17 \\
1.99\end{array}$ & - & 17 & - & - & 08 & - & \multicolumn{2}{|c|}{ Opportunity 25} \\
\hline & $\mathrm{S}$ & 5.58 & 2.42 & 5.17 & 1.42 & - & - & - & - & 08 & - & \multicolumn{2}{|c|}{ Opportunity 25} \\
\hline & & 1.38 & 1.51 & 1.53 & 1.44 & & & & & & & $\begin{array}{l}\text { Game } \\
\text { Luck }\end{array}$ & $\begin{array}{l}17 \\
17\end{array}$ \\
\hline \multirow[t]{2}{*}{ Cigar } & B & $\begin{array}{l}6.33 \\
1.15\end{array}$ & $\begin{array}{l}6.00 \\
1.21\end{array}$ & $\begin{array}{l}4.50 \\
1.62\end{array}$ & $\begin{array}{l}6.50 \\
0.80\end{array}$ & 08 & - & 08 & - & 08 & 75 & $\begin{array}{l}\text { Smoke } \\
\text { Cigarette }\end{array}$ & $\begin{array}{l}58 \\
17\end{array}$ \\
\hline & $\mathrm{S}$ & $\begin{array}{l}5.50 \\
1.62\end{array}$ & $\begin{array}{l}6.42 \\
0.79\end{array}$ & $\begin{array}{l}5.00 \\
1.48\end{array}$ & $\begin{array}{l}6.42 \\
0.79\end{array}$ & 50 & - & - & - & - & 50 & $\begin{array}{l}\text { Smoke } \\
\text { Cigarette }\end{array}$ & $\begin{array}{l}67 \\
25\end{array}$ \\
\hline \multirow[t]{2}{*}{ Circle } & B & $\begin{array}{l}6.67 \\
0.78\end{array}$ & $\begin{array}{l}5.33 \\
1.56\end{array}$ & $\begin{array}{l}4.83 \\
1.59\end{array}$ & $\begin{array}{l}5.58 \\
1.88\end{array}$ & 08 & - & 33 & - & 50 & - & $\begin{array}{l}\text { Round } \\
\text { Square }\end{array}$ & $\begin{array}{l}67 \\
17\end{array}$ \\
\hline & $\mathrm{S}$ & $\begin{array}{l}6.00 \\
0.85\end{array}$ & $\begin{array}{l}3.75 \\
1.54\end{array}$ & $\begin{array}{l}4.25 \\
2.05\end{array}$ & $\begin{array}{l}6.25 \\
0.87\end{array}$ & 75 & - & - & - & 25 & - & $\begin{array}{l}\text { Square } \\
\text { Round } \\
\text { Shape }\end{array}$ & $\begin{array}{l}33 \\
17 \\
17\end{array}$ \\
\hline \multirow[t]{2}{*}{ City } & $B$ & $\begin{array}{l}6.50 \\
1.17\end{array}$ & $\begin{array}{l}5.33 \\
1.92\end{array}$ & $\begin{array}{l}5.42 \\
1.78\end{array}$ & $\begin{array}{l}5.17 \\
1.80\end{array}$ & 17 & 25 & 08 & - & 42 & - & $\begin{array}{l}\text { Town } \\
\text { Atlanta }\end{array}$ & $\begin{array}{l}33 \\
25\end{array}$ \\
\hline & $\mathrm{S}$ & $\begin{array}{l}6.42 \\
0.67\end{array}$ & $\begin{array}{l}6.17 \\
0.83\end{array}$ & $\begin{array}{l}6.08 \\
0.90\end{array}$ & $\begin{array}{l}6.17 \\
0.72\end{array}$ & 83 & 08 & - & - & 08 & - & $\begin{array}{l}\text { State } \\
\text { Atlanta }\end{array}$ & $\begin{array}{l}25 \\
17\end{array}$ \\
\hline
\end{tabular}


APPENDIX (Continued)

\begin{tabular}{|c|c|c|c|c|c|c|c|c|c|c|c|c|c|}
\hline \multirow{2}{*}{ Noun } & \multirow{2}{*}{$\begin{array}{c}\text { Subject } \\
\text { Group }\end{array}$} & \multirow[b]{2}{*}{$\mathrm{f}$} & \multirow[b]{2}{*}{ c } & \multirow[b]{2}{*}{$\mathrm{m}$} & \multirow[b]{2}{*}{$\mathrm{i}$} & \multicolumn{6}{|c|}{ Modality (\%) } & \multirow{2}{*}{$\begin{array}{c}\text { Strongest } \\
\text { Associates }\end{array}$} & \multirow[b]{2}{*}{$\%$} \\
\hline & & & & & & $(\mathrm{v}$ & $\mathbf{a}$ & tc & ts & sp & sm) & & \\
\hline \multirow[t]{4}{*}{ Clang } & B & $\begin{array}{r}6.42 \\
0.79\end{array}$ & 6.58 & $\begin{array}{r}5.08 \\
178\end{array}$ & 6.50 & -1 & 100 & - & - & - & - & Bel1 & 50 \\
\hline & & 0.79 & 0.79 & 1.78 & 0.90 & & & & & & & Metal & 17 \\
\hline & $\mathrm{s}$ & 4.75 & 3.67 & 4.08 & 5.42 & -1 & 100 & - & - & - & - & Bel1 & 25 \\
\hline & & 1.71 & 1.83 & 1.56 & 1.56 & & & & & & & Noise & 17 \\
\hline \multirow[t]{3}{*}{ Click } & B & 6.58 & 6.17 & 5.83 & 6.42 & -1 & 100 & - & - & - & - & Noise & 42 \\
\hline & & 0.67 & 1.19 & 1.40 & 0.79 & & & & & & & Clock & 17 \\
\hline & $\mathrm{S}$ & $\begin{array}{l}4.92 \\
1.98\end{array}$ & $\begin{array}{l}5.08 \\
1.16\end{array}$ & $\begin{array}{l}4.42 \\
1.31\end{array}$ & $\begin{array}{l}5.75 \\
1.06\end{array}$ & 08 & 92 & - & - & - & - & Groups & 33 \\
\hline \multirow[t]{2}{*}{ clock } & B & $\begin{array}{l}6.83 \\
0.39\end{array}$ & $\begin{array}{l}6.58 \\
0.79\end{array}$ & $\begin{array}{l}5.67 \\
0.89\end{array}$ & $\begin{array}{l}6.58 \\
0.67\end{array}$ & - & 67 & 33 & - & - & - & Time & 50 \\
\hline & $\mathrm{S}$ & $\begin{array}{l}6.25 \\
0.97\end{array}$ & $\begin{array}{l}6.08 \\
0.79\end{array}$ & $\begin{array}{l}5.42 \\
1.31\end{array}$ & $\begin{array}{l}6.08 \\
1.16\end{array}$ & 50 & 50 & - & - & - & - & $\begin{array}{l}\text { Time } \\
\text { Tick }\end{array}$ & $\begin{array}{l}33 \\
25\end{array}$ \\
\hline \multirow[t]{4}{*}{ clown } & B & 6.42 & 5.25 & 4.92 & 5.67 & 17 & 25 & 25 & - & 17 & - & Circus & 50 \\
\hline & & 1.16 & 2.09 & 1.51 & 1.30 & & & & & & & Funny & 17 \\
\hline & & & & & & & & & & & & Laughter & 17 \\
\hline & s & $\begin{array}{l}5.67 \\
1.67\end{array}$ & $\begin{array}{l}6.08 \\
0.67\end{array}$ & $\begin{array}{l}5.42 \\
1.31\end{array}$ & $\begin{array}{l}6.33 \\
0.65\end{array}$ & 100 & $0-$ & - & - & - & - & Circus & 25 \\
\hline \multirow[t]{4}{*}{ Coin } & B & 6.75 & 6.58 & 6.25 & 6.67 & - & - & 100 & - & - & - & Money & 58 \\
\hline & & 0.43 & 0.90 & 0.13 & 0.03 & & & & & & & Penny & 17 \\
\hline & $\mathrm{S}$ & 6.50 & 6.25 & 5.42 & 6.50 & 67 & - & 33 & - & - & - & Money & 33 \\
\hline & & 0.52 & 0.97 & 1.51 & 0.67 & & & & & & & Penny & 33 \\
\hline \multirow[t]{3}{*}{ Competence } & B & 6.33 & 2.25 & 3.83 & 2.25 & - & 08 & - & - & 08 & - & Ability & 42 \\
\hline & & 0.98 & 1.60 & 1.95 & 2.30 & & & & & & & & \\
\hline & $\mathrm{S}$ & $\begin{array}{l}5.17 \\
1.58\end{array}$ & $\begin{array}{l}2.33 \\
1.50\end{array}$ & $\begin{array}{l}4.42 \\
1.31\end{array}$ & $\begin{array}{l}1.92 \\
1.56\end{array}$ & 17 & - & - & - & 17 & - & Ability & 42 \\
\hline \multirow[t]{4}{*}{ Concept } & B & 6.33 & 3.17 & 3.67 & 2.33 & 08 & - & - & - & 08 & 08 & I dea & 67 \\
\hline & & 0.89 & 2.48 & 2.15 & 2.02 & & & & & & & & \\
\hline & $\mathrm{S}$ & 5.50 & 1.83 & 3.83 & 1.58 & 08 & - & - & - & 17 & - & Idea & 58 \\
\hline & & 1.17 & 0.94 & 1.47 & 1.24 & & & & & & & & \\
\hline \multirow[t]{3}{*}{ Confidence } & B & 6.42 & 2.42 & 4.17 & 2.33 & - & 08 & - & - & - & - & Self/Self & \\
\hline & & 1.00 & 1.68 & 1.75 & 2.42 & & & & & & & Image & 17 \\
\hline & $\mathrm{S}$ & $\begin{array}{l}5.92 \\
0.67\end{array}$ & $\begin{array}{l}2.83 \\
1.64\end{array}$ & $\begin{array}{l}4.42 \\
1.88\end{array}$ & $\begin{array}{l}2.75 \\
2.30\end{array}$ & 33 & - & 08 & - & 08 & - & & \\
\hline
\end{tabular}


APPENDIX (Continued)

\begin{tabular}{|c|c|c|c|c|c|c|c|c|c|c|c|c|c|}
\hline \multirow{2}{*}{ Noun } & \multirow{2}{*}{$\begin{array}{l}\text { Subject } \\
\text { Group }\end{array}$} & \multirow[b]{2}{*}{$\mathrm{f}$} & \multirow[b]{2}{*}{ c } & \multirow[b]{2}{*}{$\mathrm{m}$} & \multirow[b]{2}{*}{$\mathrm{i}$} & \multicolumn{6}{|c|}{ Modality (\%) } & \multirow{2}{*}{$\begin{array}{c}\text { Strongest } \\
\text { Associates }\end{array}$} & \multirow[b]{2}{*}{$\%$} \\
\hline & & & & & & $(\mathrm{v}$ & $a t$ & tc & ts & sp & $\mathrm{sm})$ & & \\
\hline Conversation & B & $\begin{array}{l}6.75 \\
0.45\end{array}$ & $\begin{array}{l}6.08 \\
1.24\end{array}$ & $\begin{array}{l}5.25 \\
1.60\end{array}$ & $\begin{array}{l}6.00 \\
1.48\end{array}$ & - & 100 & - & - & - & - & Talking & 83 \\
\hline & s & $\begin{array}{l}5.92 \\
0.90\end{array}$ & $\begin{array}{l}3.92 \\
2.15\end{array}$ & $\begin{array}{l}5.33 \\
1.37\end{array}$ & $\begin{array}{l}4.92 \\
1.88\end{array}$ & 25 & $67-$ & - & - & 08 & - & Talking & 67 \\
\hline \multirow[t]{2}{*}{ Cooperation } & B & $\begin{array}{l}6.58 \\
0.79\end{array}$ & $\begin{array}{l}2.92 \\
1.73\end{array}$ & $\begin{array}{l}4.33 \\
1.78\end{array}$ & $\begin{array}{l}2.33 \\
2.42\end{array}$ & - & $08-$ & - & - & 08 & - & \multicolumn{2}{|c|}{$\begin{array}{l}\text { Togetherness } 25 \\
\text { Going along } 17\end{array}$} \\
\hline & $\mathrm{s}$ & $\begin{array}{l}5.58 \\
1.08\end{array}$ & $\begin{array}{l}2.50 \\
1.57\end{array}$ & $\begin{array}{l}5.00 \\
1.86\end{array}$ & $\begin{array}{l}2.58 \\
2.07\end{array}$ & 42 & - & - & - & 08 & - & Help & 42 \\
\hline \multirow[t]{2}{*}{ Corner } & B & $\begin{array}{l}6.25 \\
0.75\end{array}$ & $\begin{array}{l}4.75 \\
1.82\end{array}$ & $\begin{array}{l}3.83 \\
1.90\end{array}$ & $\begin{array}{l}5.17 \\
1.80\end{array}$ & 17 & -2 & 25 & - & 50 & - & Street & 25 \\
\hline & $\mathrm{s}$ & $\begin{array}{l}5.58 \\
1.38\end{array}$ & $\begin{array}{l}5.42 \\
1.31\end{array}$ & $\begin{array}{l}4.42 \\
1.73\end{array}$ & $\begin{array}{l}5.25 \\
1.29\end{array}$ & 100 & - & - & - & - & - & $\begin{array}{l}\text { Edge } \\
\text { Room }\end{array}$ & $\begin{array}{l}17 \\
17\end{array}$ \\
\hline \multirow[t]{2}{*}{ Cottage } & B & $\begin{array}{l}6.25 \\
1.06\end{array}$ & $\begin{array}{l}5.83 \\
1.27\end{array}$ & $\begin{array}{l}4.08 \\
1.88\end{array}$ & $\begin{array}{l}5.58 \\
1.51\end{array}$ & 17 & -4 & 42 & - & 42 & - & $\begin{array}{l}\text { House } \\
\text { Home }\end{array}$ & $\begin{array}{l}42 \\
17\end{array}$ \\
\hline & $s$ & $\begin{array}{l}5.42 \\
1.44\end{array}$ & $\begin{array}{l}5.75 \\
0.97\end{array}$ & $\begin{array}{l}4.17 \\
1.75\end{array}$ & $\begin{array}{l}5.83 \\
1.19\end{array}$ & 100 & - & - & - & - & - & $\begin{array}{l}\text { House } \\
\text { Cheese }\end{array}$ & $\begin{array}{l}58 \\
17\end{array}$ \\
\hline \multirow[t]{2}{*}{ Cough } & B & $\begin{array}{l}6.67 \\
0.65\end{array}$ & $\begin{array}{l}6.25 \\
1.06\end{array}$ & $\begin{array}{l}5.83 \\
0.83\end{array}$ & $\begin{array}{l}5.75 \\
2.09\end{array}$ & -6 & 671 & 17 & - & - & - & $\begin{array}{l}\text { Cold } \\
\text { Sneeze }\end{array}$ & $\begin{array}{l}50 \\
17\end{array}$ \\
\hline & $\mathrm{s}$ & $\begin{array}{l}6.08 \\
1.00\end{array}$ & $\begin{array}{l}5.75 \\
1.14\end{array}$ & $\begin{array}{l}5.42 \\
1.51\end{array}$ & $\begin{array}{l}6.08 \\
0.79\end{array}$ & 08 & 583 & 33 & - & - & - & $\begin{array}{l}\text { Cold } \\
\text { Sneeze }\end{array}$ & $\begin{array}{l}33 \\
25\end{array}$ \\
\hline \multirow[t]{2}{*}{ Custom } & B & $\begin{array}{l}6.25 \\
1.06\end{array}$ & $\begin{array}{l}2.67 \\
2.15\end{array}$ & $\begin{array}{l}3.92 \\
1.88\end{array}$ & $\begin{array}{l}1.83 \\
1.99\end{array}$ & 08 & - & - & - & - & - & $\begin{array}{l}\text { Traditions } \\
\text { Habit }\end{array}$ & $\begin{array}{l}50 \\
17\end{array}$ \\
\hline & $\mathrm{s}$ & $\begin{array}{l}5.08 \\
1.38\end{array}$ & $\begin{array}{l}2.83 \\
1.34\end{array}$ & $\begin{array}{l}3.17 \\
1.47\end{array}$ & $\begin{array}{l}2.83 \\
2.17\end{array}$ & 42 & $08-$ & - & - & - & - & & \\
\hline \multirow[t]{2}{*}{ Dawn } & B & $\begin{array}{l}6.42 \\
1.51\end{array}$ & $\begin{array}{l}5.33 \\
1.87\end{array}$ & $\begin{array}{l}4.92 \\
1.83\end{array}$ & $\begin{array}{l}4.33 \\
2.31\end{array}$ & 25 & $08-$ & - & - & 33 & - & $\begin{array}{l}\text { Early } \\
\text { Morning }\end{array}$ & $\begin{array}{l}25 \\
17\end{array}$ \\
\hline & S & $\begin{array}{l}5.92 \\
1.24\end{array}$ & $\begin{array}{l}5.42 \\
1.24\end{array}$ & $\begin{array}{l}5.50 \\
1.00\end{array}$ & $\begin{array}{l}5.75 \\
1.14\end{array}$ & 100 & - & - & - & - & - & $\begin{array}{l}\text { Day } \\
\text { Daylight } \\
\text { Morning }\end{array}$ & $\begin{array}{l}17 \\
17 \\
17\end{array}$ \\
\hline \multirow[t]{2}{*}{ Daybreak } & B & $\begin{array}{l}6.17 \\
1.27\end{array}$ & $\begin{array}{l}4.67 \\
2.06\end{array}$ & $\begin{array}{l}3.42 \\
2.02\end{array}$ & $\begin{array}{l}4.67 \\
2.19\end{array}$ & 33 & $25-$ & - & - & 08 & 08 & $\begin{array}{l}\text { Dawn } \\
\text { Morning }\end{array}$ & $\begin{array}{l}50 \\
17\end{array}$ \\
\hline & s & $\begin{array}{l}5.33 \\
1.23\end{array}$ & $\begin{array}{l}5.58 \\
1.16\end{array}$ & $\begin{array}{l}4.75 \\
1.66\end{array}$ & $\begin{array}{l}5.42 \\
1.31\end{array}$ & 100 & - & - & - & - & - & $\begin{array}{l}\text { Morning } \\
\text { Sunrise/se }\end{array}$ & $\begin{array}{r}25 \\
t 25\end{array}$ \\
\hline
\end{tabular}


APPENDIX (Continued)

\begin{tabular}{|c|c|c|c|c|c|c|c|c|c|c|c|c|c|}
\hline \multirow[b]{2}{*}{ Noun } & \multirow{2}{*}{$\begin{array}{l}\text { Subject } \\
\text { Group }\end{array}$} & \multirow[b]{2}{*}{$\mathrm{f}$} & \multirow[b]{2}{*}{ c } & \multirow[b]{2}{*}{$\mathrm{m}$} & \multirow[b]{2}{*}{$\mathrm{i}$} & \multicolumn{6}{|c|}{ Modality (\%) } & \multirow{2}{*}{$\begin{array}{c}\text { Strongest } \\
\text { Associates }\end{array}$} & \multirow[b]{2}{*}{$\%$} \\
\hline & & & & & & $(\mathrm{v}$ & a & tc & ts & $\mathrm{sp}$ & sm) & & \\
\hline \multirow[t]{2}{*}{ Daylight } & B & $\begin{array}{l}6.58 \\
1.16\end{array}$ & $\begin{array}{l}5.33 \\
2.06\end{array}$ & $\begin{array}{l}4.67 \\
1.78\end{array}$ & $\begin{array}{l}5.00 \\
2.30\end{array}$ & 42 & 08 & 08 & - & 17 & - & $\begin{array}{l}\text { Dark } \\
\text { Dawn }\end{array}$ & $\begin{array}{l}25 \\
17\end{array}$ \\
\hline & $\mathrm{s}$ & $\begin{array}{l}5.75 \\
1.48\end{array}$ & $\begin{array}{l}5.58 \\
1.38\end{array}$ & $\begin{array}{l}5.50 \\
1.09\end{array}$ & $\begin{array}{l}6.00 \\
0.95\end{array}$ & 92 & - & 08 & - & - & - & $\begin{array}{l}\text { Living } \\
\text { Night } \\
\text { Sunshine }\end{array}$ & $\begin{array}{l}17 \\
17 \\
17\end{array}$ \\
\hline \multirow[t]{2}{*}{ Democracy } & B & $\begin{array}{l}6.42 \\
0.79\end{array}$ & $\begin{array}{l}2.50 \\
1.83\end{array}$ & $\begin{array}{l}4.50 \\
1.73\end{array}$ & $\begin{array}{l}1.83 \\
1.40\end{array}$ & - & 08 & - & - & 17 & - & $\begin{array}{l}\text { Government } \\
\text { America }\end{array}$ & $\begin{array}{l}42 \\
25\end{array}$ \\
\hline & $\mathrm{s}$ & $\begin{array}{l}5.58 \\
1.31\end{array}$ & $\begin{array}{l}2.67 \\
0.98\end{array}$ & $\begin{array}{l}5.08 \\
1.44\end{array}$ & $\begin{array}{l}2.50 \\
2.15\end{array}$ & 25 & - & - & - & 17 & - & $\begin{array}{l}\text { America } \\
\text { Government }\end{array}$ & $\begin{array}{l}17 \\
17\end{array}$ \\
\hline \multirow[t]{2}{*}{ Devotion } & B & $\begin{array}{l}6.75 \\
0.45\end{array}$ & $\begin{array}{l}2.58 \\
1.62\end{array}$ & $\begin{array}{l}4.17 \\
1.99\end{array}$ & $\begin{array}{l}2.17 \\
2.04\end{array}$ & - & 08 & - & - & 17 & - & $\begin{array}{l}\text { Devoted } \\
\text { Loyalty } \\
\text { Prayer }\end{array}$ & $\begin{array}{l}17 \\
17 \\
17\end{array}$ \\
\hline & S & $\begin{array}{l}5.08 \\
1.24\end{array}$ & $\begin{array}{l}2.83 \\
1.99\end{array}$ & $\begin{array}{l}4.83 \\
1.80\end{array}$ & $\begin{array}{l}2.25 \\
2.30\end{array}$ & 08 & - & - & - & 17 & - & Love & 42 \\
\hline \multirow[t]{2}{*}{ Diamond } & B & $\begin{array}{l}6.42 \\
0.67\end{array}$ & $\begin{array}{l}5.83 \\
1.75\end{array}$ & $\begin{array}{l}4.92 \\
1.51\end{array}$ & $\begin{array}{l}6.00 \\
1.28\end{array}$ & 08 & - & 83 & - & 08 & - & $\begin{array}{l}\text { Ring } \\
\text { Jewel } \\
\text { Jewel ry }\end{array}$ & $\begin{array}{l}33 \\
17 \\
17\end{array}$ \\
\hline & $\mathrm{s}$ & $\begin{array}{l}5.67 \\
1.37\end{array}$ & $\begin{array}{l}6.25 \\
0.75\end{array}$ & $\begin{array}{l}4.92 \\
1.83\end{array}$ & $\begin{array}{l}6.17 \\
0.94\end{array}$ & 83 & - & 17 & - & - & - & $\begin{array}{l}\text { Ring } \\
\text { Stone }\end{array}$ & $\begin{array}{l}17 \\
17\end{array}$ \\
\hline \multirow[t]{2}{*}{ Discretion } & B & $\begin{array}{l}6.08 \\
1.00\end{array}$ & $\begin{array}{l}2.75 \\
1.91\end{array}$ & $\begin{array}{l}3.08 \\
2.02\end{array}$ & $\begin{array}{l}2.33 \\
2.19\end{array}$ & - & 17 & - & - & 08 & - & $\begin{array}{l}\text { Caut ious } \\
\text { Judgment }\end{array}$ & $\begin{array}{l}25 \\
17\end{array}$ \\
\hline & $\mathrm{s}$ & $\begin{array}{l}4.83 \\
1.40\end{array}$ & $\begin{array}{l}2.08 \\
1.00\end{array}$ & $\begin{array}{l}4.17 \\
1.75\end{array}$ & $\begin{array}{l}1.50 \\
1.17\end{array}$ & 17 & - & - & - & - & - & $\begin{array}{l}\text { Parental } \\
\text { Secrecy }\end{array}$ & $\begin{array}{l}17 \\
17\end{array}$ \\
\hline \multirow[t]{2}{*}{ Doll } & B & $\begin{array}{l}6.83 \\
0.39\end{array}$ & $\begin{array}{l}6.50 \\
0.90\end{array}$ & $\begin{array}{l}5.67 \\
1.30\end{array}$ & $\begin{array}{l}6.58 \\
0.79\end{array}$ & - & - & 92 & - & 08 & - & $\begin{array}{l}\text { Plaything } \\
\text { House }\end{array}$ & $\begin{array}{l}33 \\
25\end{array}$ \\
\hline & $\mathrm{s}$ & $\begin{array}{l}5.67 \\
1.50\end{array}$ & $\begin{array}{l}5.92 \\
1.08\end{array}$ & $\begin{array}{l}5.17 \\
1.80\end{array}$ & $\begin{array}{l}5.92 \\
1.16\end{array}$ & 92 & - & 08 & - & - & - & $\begin{array}{l}\text { House } \\
\text { Child } \\
\text { Toy }\end{array}$ & $\begin{array}{l}25 \\
17 \\
17\end{array}$ \\
\hline \multirow[t]{2}{*}{ Dragon } & B & $\begin{array}{l}6.00 \\
1.48\end{array}$ & $\begin{array}{l}4.58 \\
2.27\end{array}$ & $\begin{array}{l}3.33 \\
1.97\end{array}$ & $\begin{array}{l}3.50 \\
2.15\end{array}$ & 25 & 17 & 17 & - & 17 & - & $\begin{array}{l}\text { Fire } \\
\text { Monster }\end{array}$ & $\begin{array}{l}33 \\
25\end{array}$ \\
\hline & $\mathrm{s}$ & $\begin{array}{l}4.83 \\
1.80\end{array}$ & $\begin{array}{l}4.33 \\
1.92\end{array}$ & $\begin{array}{l}4.75 \\
1.48\end{array}$ & $\begin{array}{l}5.50 \\
1.24\end{array}$ & 100 & - & - & - & - & - & Fire & 33 \\
\hline Echo & B & $\begin{array}{l}6.33 \\
1.15\end{array}$ & $\begin{array}{l}6.42 \\
0.79\end{array}$ & $\begin{array}{l}4.17 \\
1.90\end{array}$ & $\begin{array}{l}5.75 \\
1.66\end{array}$ & - & 92 & - & - & 08 & - & $\begin{array}{l}\text { Sound/Noise } \\
\text { Mountains }\end{array}$ & $\begin{array}{r}25 \\
17\end{array}$ \\
\hline
\end{tabular}


APPENDIX (Continued)

\begin{tabular}{|c|c|c|c|c|c|c|c|c|c|c|c|c|c|}
\hline \multirow[b]{2}{*}{ Noun } & \multirow{2}{*}{$\begin{array}{c}\text { Subject } \\
\text { Group }\end{array}$} & \multirow[b]{2}{*}{$\mathrm{f}$} & \multirow[b]{2}{*}{$\mathrm{c}$} & \multirow[b]{2}{*}{$\mathrm{m}$} & \multirow[b]{2}{*}{$\mathrm{i}$} & \multicolumn{6}{|c|}{ Modality (\%) } & \multirow{2}{*}{$\begin{array}{c}\text { Strongest } \\
\text { Associates }\end{array}$} & \multirow[b]{2}{*}{$\%$} \\
\hline & & & & & & $(\mathrm{v}$ & a & tc & ts & $\mathrm{sp}$ & $\mathrm{sm})$ & & \\
\hline & $\mathrm{S}$ & $\begin{array}{l}5.00 \\
1.71\end{array}$ & $\begin{array}{l}4.83 \\
1.90\end{array}$ & $\begin{array}{l}3.92 \\
1.68\end{array}$ & $\begin{array}{l}5.25 \\
1.29\end{array}$ & 08 & 83 & - & - & 08 & - & Canyon & 33 \\
\hline \multirow[t]{2}{*}{ Elephant } & B & $\begin{array}{l}6.17 \\
1.11\end{array}$ & $\begin{array}{l}6.25 \\
1.29\end{array}$ & $\begin{array}{l}4.92 \\
1.83\end{array}$ & $\begin{array}{l}6.08 \\
1.24\end{array}$ & 08 & 17 & 58 & - & 17 & - & $\begin{array}{l}\text { Animal } \\
\text { Trunk } \\
\text { Zoo }\end{array}$ & $\begin{array}{l}25 \\
17 \\
17\end{array}$ \\
\hline & $S$ & $\begin{array}{l}5.83 \\
1.27\end{array}$ & $\begin{array}{l}6.33 \\
0.77\end{array}$ & $\begin{array}{l}4.58 \\
2.19\end{array}$ & $\begin{array}{l}6.33 \\
0.65\end{array}$ & 100 & - & - & - & - & - & $\begin{array}{l}\text { Big } \\
\text { Large } \\
\text { Tusk }\end{array}$ & $\begin{array}{l}17 \\
17 \\
17\end{array}$ \\
\hline \multirow[t]{2}{*}{ Engine } & $\mathrm{B}$ & $\begin{array}{l}6.17 \\
1.47\end{array}$ & $\begin{array}{l}6.00 \\
1.60\end{array}$ & $\begin{array}{l}5.08 \\
1.38\end{array}$ & $\begin{array}{l}5.25 \\
1.91\end{array}$ & 08 & 67 & 17 & - & - & - & $\begin{array}{l}\text { Car } \\
\text { Motor } \\
\text { Train }\end{array}$ & $\begin{array}{l}42 \\
17 \\
17\end{array}$ \\
\hline & $\mathrm{S}$ & $\begin{array}{l}5.67 \\
1.56\end{array}$ & $\begin{array}{l}6.33 \\
0.78\end{array}$ & $\begin{array}{l}5.25 \\
1.36\end{array}$ & $\begin{array}{l}5.42 \\
1.08\end{array}$ & 50 & 50 & - & - & - & - & $\begin{array}{l}\text { Car } \\
\text { Roar }\end{array}$ & $\begin{array}{l}25 \\
17\end{array}$ \\
\hline \multirow[t]{2}{*}{ Essence } & B & $\begin{array}{l}5.92 \\
1.31\end{array}$ & $\begin{array}{l}2.75 \\
2.14\end{array}$ & $\begin{array}{l}2.75 \\
1.71\end{array}$ & $\begin{array}{l}1.75 \\
1.48\end{array}$ & - & 08 & - & - & 08 & 08 & Perfume & 17 \\
\hline & $\mathbf{S}$ & $\begin{array}{l}4.50 \\
1.73\end{array}$ & $\begin{array}{l}2.50 \\
1.09\end{array}$ & $\begin{array}{l}3.42 \\
1.62\end{array}$ & $\begin{array}{l}3.25 \\
2.53\end{array}$ & 08 & - & - & - & 08 & 33 & $\begin{array}{l}\text { Smel1 } \\
\text { Experience }\end{array}$ & $\begin{array}{l}33 \\
17\end{array}$ \\
\hline \multirow[t]{2}{*}{ Explosion } & B & $\begin{array}{l}6.50 \\
0.90\end{array}$ & $\begin{array}{l}6.58 \\
0.79\end{array}$ & $\begin{array}{l}4.58 \\
1.68\end{array}$ & $\begin{array}{l}6.00 \\
1.21\end{array}$ & - & 92 & 08 & - & - & - & $\begin{array}{l}\text { Fire } \\
\text { Noise/Sound }\end{array}$ & $\begin{array}{r}17 \\
d 17\end{array}$ \\
\hline & $S$ & $\begin{array}{l}5.58 \\
1.24\end{array}$ & $\begin{array}{l}5.83 \\
1.27\end{array}$ & $\begin{array}{l}5.25 \\
1.60\end{array}$ & $\begin{array}{l}6.08 \\
1.00\end{array}$ & 33 & 67 & - & - & - & - & $\begin{array}{l}\text { Bomb } \\
\text { Boom }\end{array}$ & $\begin{array}{l}42 \\
17\end{array}$ \\
\hline \multirow[t]{2}{*}{ Fallacy } & $B$ & $\begin{array}{l}5.83 \\
1.19\end{array}$ & $\begin{array}{l}2.83 \\
2.17\end{array}$ & $\begin{array}{l}3.08 \\
1.68\end{array}$ & $\begin{array}{l}1.67 \\
1.61\end{array}$ & - & 08 & - & - & - & - & Falsehood & 25 \\
\hline & $S$ & $\begin{array}{l}4.33 \\
1.72\end{array}$ & $\begin{array}{l}1.75 \\
0.87\end{array}$ & $\begin{array}{l}3.42 \\
1.51\end{array}$ & $\begin{array}{l}1.33 \\
0.89\end{array}$ & - & 08 & - & - & - & - & $\begin{array}{l}\text { False } \\
\text { Wrong }\end{array}$ & $\begin{array}{l}58 \\
17\end{array}$ \\
\hline \multirow[t]{2}{*}{ Fate } & B & $\begin{array}{l}6.08 \\
1.00\end{array}$ & $\begin{array}{l}2.25 \\
1.76\end{array}$ & $\begin{array}{l}2.67 \\
1.50\end{array}$ & $\begin{array}{l}1.67 \\
1.61\end{array}$ & - & - & - & - & 08 & - & Destiny & 42 \\
\hline & $S$ & $\begin{array}{l}5.42 \\
1.16\end{array}$ & $\begin{array}{l}2.17 \\
1.27\end{array}$ & $\begin{array}{l}3.75 \\
1.82\end{array}$ & $\begin{array}{l}1.17 \\
0.58\end{array}$ & - & - & - & - & 08 & - & Destiny & 42 \\
\hline \multirow[t]{2}{*}{ Flag } & B & $\begin{array}{l}6.58 \\
0.90\end{array}$ & $\begin{array}{l}5.58 \\
1.16\end{array}$ & $\begin{array}{l}5.33 \\
1.15\end{array}$ & $\begin{array}{l}6.00 \\
1.28\end{array}$ & 25 & - & 58 & - & 17 & - & American & 33 \\
\hline & $\mathrm{S}$ & $\begin{array}{l}6.00 \\
1.21\end{array}$ & $\begin{array}{l}5.83 \\
1.19\end{array}$ & $\begin{array}{l}4.92 \\
1.44\end{array}$ & $\begin{array}{l}5.67 \\
0.89\end{array}$ & 92 & - & 08 & - & - & - & $\begin{array}{l}\text { American } \\
\text { Pole }\end{array}$ & $\begin{array}{l}25 \\
17\end{array}$ \\
\hline
\end{tabular}


APPENDIX (Continued)

\begin{tabular}{|c|c|c|c|c|c|c|c|c|c|c|c|c|c|}
\hline \multirow[b]{2}{*}{ Noun } & \multirow{2}{*}{$\begin{array}{l}\text { Subject } \\
\text { Group }\end{array}$} & \multirow[b]{2}{*}{$\mathrm{f}$} & \multirow[b]{2}{*}{ c } & \multirow[b]{2}{*}{$\mathrm{m}$} & \multirow[b]{2}{*}{$\mathrm{i}$} & \multicolumn{6}{|c|}{ Modality (\%) } & \multirow{2}{*}{$\begin{array}{c}\text { Strongest } \\
\text { Associates }\end{array}$} & \multirow[b]{2}{*}{$\%$} \\
\hline & & & & & & $(\mathrm{v}$ & a & tc & ts & $\mathrm{sp}$ & sm) & & \\
\hline \multirow[t]{2}{*}{ Flower } & B & $\begin{array}{l}6.67 \\
0.65\end{array}$ & $\begin{array}{l}6.17 \\
0.83\end{array}$ & $\begin{array}{l}5.83 \\
0.94\end{array}$ & $\begin{array}{l}6.42 \\
0.67\end{array}$ & - & -5 & 58 & - & - & 42 & $\begin{array}{l}\text { Bloom } \\
\text { Pretty }\end{array}$ & $\begin{array}{l}17 \\
17\end{array}$ \\
\hline & s & $\begin{array}{l}6.08 \\
1.44\end{array}$ & $\begin{array}{l}6.25 \\
1.06\end{array}$ & $\begin{array}{l}5.92 \\
1.24\end{array}$ & $\begin{array}{l}6.33 \\
0.89\end{array}$ & 83 & - & - & - & - & 17 & $\begin{array}{l}\text { Rose } \\
\text { Plant } \\
\text { Pretty }\end{array}$ & $\begin{array}{l}25 \\
17 \\
17\end{array}$ \\
\hline \multirow[t]{2}{*}{ Fork } & B & $\begin{array}{l}7.00 \\
0.00\end{array}$ & $\begin{array}{l}6.58 \\
0.79\end{array}$ & $\begin{array}{l}6.25 \\
1.14\end{array}$ & $\begin{array}{l}6.75 \\
0.62\end{array}$ & - & $-s$ & 92 & - & 08 & - & $\begin{array}{l}\text { Eat/Eating } \\
\text { Knife } \\
\text { Utensil }\end{array}$ & $\begin{array}{l}33 \\
17 \\
17\end{array}$ \\
\hline & $\mathrm{s}$ & $\begin{array}{l}6.67 \\
0.65\end{array}$ & $\begin{array}{l}6.67 \\
0.65\end{array}$ & $\begin{array}{l}5.17 \\
1.27\end{array}$ & $\begin{array}{l}6.33 \\
0.89\end{array}$ & 83 & - & 08 & 08 & - & - & $\begin{array}{l}\text { Spoon } \\
\text { Utensil }\end{array}$ & $\begin{array}{l}33 \\
25\end{array}$ \\
\hline \multirow[t]{2}{*}{ Galaxy } & B & $\begin{array}{l}5.92 \\
1.24\end{array}$ & $\begin{array}{l}4.25 \\
2.38\end{array}$ & $\begin{array}{l}3.42 \\
1.98\end{array}$ & $\begin{array}{l}3.67 \\
2.10\end{array}$ & 33 & - & 08 & - & 33 & - & $\begin{array}{l}\text { Stars } \\
\text { Space }\end{array}$ & $\begin{array}{l}42 \\
17\end{array}$ \\
\hline & s & $\begin{array}{l}5.25 \\
1.54\end{array}$ & $\begin{array}{l}5.08 \\
1.24\end{array}$ & $\begin{array}{l}4.92 \\
1.98\end{array}$ & $\begin{array}{l}5.67 \\
1.30\end{array}$ & 50 & - & - & - & 50 & - & $\begin{array}{l}\text { Star } \\
\text { Universe }\end{array}$ & $\begin{array}{l}25 \\
25\end{array}$ \\
\hline \multirow[t]{2}{*}{ Glory } & B & $\begin{array}{l}6.50 \\
0.52\end{array}$ & $\begin{array}{l}2.92 \\
1.98\end{array}$ & $\begin{array}{l}3.58 \\
2.07\end{array}$ & $\begin{array}{l}2.58 \\
2.39\end{array}$ & 08 & 08 & 08 & - & 08 & - & Honor & 25 \\
\hline & S & $\begin{array}{l}5.17 \\
1.34\end{array}$ & $\begin{array}{l}2.83 \\
1.75\end{array}$ & $\begin{array}{l}4.75 \\
1.48\end{array}$ & $\begin{array}{l}2.17 \\
2.04\end{array}$ & 33 & - & - & - & - & - & $\begin{array}{l}\text { God } \\
\text { Honor }\end{array}$ & $\begin{array}{l}17 \\
17\end{array}$ \\
\hline \multirow[t]{2}{*}{ Goblet } & B & $\begin{array}{l}6.42 \\
1.00\end{array}$ & $\begin{array}{l}6.58 \\
0.67\end{array}$ & $\begin{array}{l}4.92 \\
1.98\end{array}$ & $\begin{array}{l}6.25 \\
1.36\end{array}$ & - & - & 83 & - & 17 & - & $\begin{array}{l}\text { Glass } \\
\text { Drink } \\
\text { Wine }\end{array}$ & $\begin{array}{l}42 \\
17 \\
17\end{array}$ \\
\hline & S & $\begin{array}{l}4.75 \\
1.54\end{array}$ & $\begin{array}{l}5.83 \\
1.19\end{array}$ & $\begin{array}{l}3.75 \\
1.48\end{array}$ & $\begin{array}{l}5.08 \\
1.38\end{array}$ & 92 & - & 08 & - & - & - & $\begin{array}{l}\text { Wine } \\
\text { Glass }\end{array}$ & $\begin{array}{l}33 \\
17\end{array}$ \\
\hline \multirow[t]{2}{*}{ Gong } & B & $\begin{array}{l}6.33 \\
0.98\end{array}$ & $\begin{array}{l}6.50 \\
1.00\end{array}$ & $\begin{array}{l}5.00 \\
1.91\end{array}$ & $\begin{array}{l}6.33 \\
1.23\end{array}$ & - & 100 & $0-$ & - & - & - & $\begin{array}{l}\text { Bell } \\
\text { Noise }\end{array}$ & $\begin{array}{l}25 \\
25\end{array}$ \\
\hline & $\mathrm{s}$ & $\begin{array}{l}4.92 \\
1.62\end{array}$ & $\begin{array}{l}5.08 \\
1.38\end{array}$ & $\begin{array}{l}3.50 \\
0.67\end{array}$ & $\begin{array}{l}5.00 \\
1.35\end{array}$ & 17 & 83 & - & - & - & - & $\begin{array}{l}\text { Show } \\
\text { Bang }\end{array}$ & $\begin{array}{l}50 \\
17\end{array}$ \\
\hline \multirow[t]{2}{*}{ Green } & B & $\begin{array}{l}6.33 \\
1.72\end{array}$ & $\begin{array}{l}4.25 \\
2.38\end{array}$ & $\begin{array}{l}4.33 \\
2.02\end{array}$ & $\begin{array}{l}3.42 \\
2.50\end{array}$ & 42 & - & 08 & - & 08 & - & $\begin{array}{l}\text { Grass } \\
\text { Color }\end{array}$ & $\begin{array}{l}58 \\
17\end{array}$ \\
\hline & $\mathrm{s}$ & $\begin{array}{l}6.08 \\
0.79\end{array}$ & $\begin{array}{l}5.00 \\
2.22\end{array}$ & $\begin{array}{l}5.83 \\
1.11\end{array}$ & $\begin{array}{l}5.92 \\
1.31\end{array}$ & 100 & - & - & - & - & - & $\begin{array}{l}\text { Blue } \\
\text { Grass }\end{array}$ & $\begin{array}{l}25 \\
25\end{array}$ \\
\hline \multirow[t]{2}{*}{ Groan } & B & $\begin{array}{l}6.42 \\
0.90\end{array}$ & $\begin{array}{l}6.33 \\
0.78\end{array}$ & $\begin{array}{l}4.00 \\
1.76\end{array}$ & $\begin{array}{l}5.92 \\
1.56\end{array}$ & - & 100 & - & - & - & - & Moan & 17 \\
\hline & s & $\begin{array}{l}5.50 \\
1.38\end{array}$ & $\begin{array}{l}5.25 \\
1.14\end{array}$ & $\begin{array}{l}4.50 \\
1.17\end{array}$ & $\begin{array}{l}4.92 \\
1.44\end{array}$ & 33 & 67 & - & - & - & - & $\begin{array}{l}\text { Moan } \\
\text { Pain }\end{array}$ & $\begin{array}{l}25 \\
17\end{array}$ \\
\hline
\end{tabular}




\begin{tabular}{|c|c|c|c|c|c|c|c|c|c|c|c|c|c|}
\hline \multirow[b]{2}{*}{ Noun } & \multirow{2}{*}{$\begin{array}{l}\text { Subject } \\
\text { Group }\end{array}$} & \multirow[b]{2}{*}{$\mathrm{f}$} & \multirow[b]{2}{*}{$\mathrm{c}$} & \multirow[b]{2}{*}{$\mathrm{m}$} & \multirow[b]{2}{*}{$\mathrm{i}$} & \multicolumn{6}{|c|}{ Modality (\%) } & \multirow{2}{*}{$\begin{array}{l}\text { Strongest } \\
\text { Associates }\end{array}$} & \multirow[b]{2}{*}{$\%$} \\
\hline & & & & & & $(\mathrm{v}$ & $\mathrm{a}$ & tc & ts & $\mathrm{sp}$ & $\mathrm{sm})$ & & \\
\hline Growl & B & $\begin{array}{l}6.83 \\
0.39\end{array}$ & $\begin{array}{l}6.42 \\
0.90\end{array}$ & $\begin{array}{l}5.67 \\
1.23\end{array}$ & $\begin{array}{l}6.67 \\
0.65\end{array}$ & - & 100 & - & - & - & - & $\begin{array}{l}\text { Dog } \\
\text { Animal }\end{array}$ & $\begin{array}{l}33 \\
17\end{array}$ \\
\hline \multirow{3}{*}{ Gun } & $\mathrm{S}$ & $\begin{array}{l}5.00 \\
1.71\end{array}$ & $\begin{array}{l}5.00 \\
1.60\end{array}$ & $\begin{array}{l}4.42 \\
1.56\end{array}$ & $\begin{array}{l}5.75 \\
1.14\end{array}$ & - & 100 & - & - & - & - & Dog & 33 \\
\hline & B & $\begin{array}{l}6.42 \\
1.00\end{array}$ & $\begin{array}{l}6.33 \\
1.07\end{array}$ & $\begin{array}{l}5.25 \\
1.60\end{array}$ & $\begin{array}{l}6.17 \\
1.03\end{array}$ & 08 & 67 & 25 & - & - & - & $\begin{array}{l}\text { Bullet } \\
\text { Shoot } \\
\text { Weapon }\end{array}$ & $\begin{array}{l}17 \\
17 \\
17\end{array}$ \\
\hline & $\mathrm{S}$ & $\begin{array}{l}6.08 \\
0.90\end{array}$ & $\begin{array}{l}6.42 \\
0.51\end{array}$ & $\begin{array}{l}5.08 \\
1.38\end{array}$ & $\begin{array}{l}6.25 \\
0.97\end{array}$ & 67 & 25 & 08 & - & - & - & $\begin{array}{l}\text { Shoot } \\
\text { Weapon }\end{array}$ & $\begin{array}{l}33 \\
17\end{array}$ \\
\hline \multirow[t]{2}{*}{ Harp } & B & $\begin{array}{l}6.33 \\
1.15\end{array}$ & $\begin{array}{l}6.08 \\
1.44\end{array}$ & $\begin{array}{l}4.75 \\
1.76\end{array}$ & $\begin{array}{l}6.25 \\
1.06\end{array}$ & - & 92 & 08 & - & - & - & $\begin{array}{l}\text { Instrument } \\
\text { Music } \\
\text { String }\end{array}$ & $\begin{array}{l}33 \\
17 \\
17\end{array}$ \\
\hline & $\mathrm{s}$ & $\begin{array}{l}4.75 \\
1.54\end{array}$ & $\begin{array}{l}5.50 \\
1.24\end{array}$ & $\begin{array}{l}4.83 \\
1.40\end{array}$ & $\begin{array}{l}5.33 \\
1.30\end{array}$ & 42 & 58 & - & - & - & - & $\begin{array}{l}\text { Instrument } \\
\text { Ange } 1\end{array}$ & $\begin{array}{l}33 \\
17\end{array}$ \\
\hline \multirow[t]{2}{*}{ Headlight } & B & $\begin{array}{l}6.50 \\
0.90\end{array}$ & $\begin{array}{l}5.58 \\
1.78\end{array}$ & $\begin{array}{l}4.67 \\
1.87\end{array}$ & $\begin{array}{l}5.58 \\
1.51\end{array}$ & 50 & - & 33 & - & 17 & - & Car & 67 \\
\hline & $\mathrm{S}$ & $\begin{array}{l}5.83 \\
1.11\end{array}$ & $\begin{array}{l}6.33 \\
0.89\end{array}$ & $\begin{array}{l}4.50 \\
2.02\end{array}$ & $\begin{array}{l}6.25 \\
0.75\end{array}$ & 100 & - & - & - & - & - & Car & 58 \\
\hline \multirow[t]{2}{*}{$\mathrm{Hi} 1 \mathrm{I}$} & $\mathrm{B}$ & $\begin{array}{l}6.42 \\
0.79\end{array}$ & $\begin{array}{l}5.75 \\
1.54\end{array}$ & $\begin{array}{l}4.75 \\
1.76\end{array}$ & $\begin{array}{l}5.17 \\
1.75\end{array}$ & 08 & - & 25 & - & 58 & - & $\begin{array}{l}\text { Mountain } \\
\text { High }\end{array}$ & $\begin{array}{l}25 \\
25\end{array}$ \\
\hline & $\mathrm{S}$ & $\begin{array}{l}6.00 \\
1.04\end{array}$ & $\begin{array}{l}5.67 \\
1.37\end{array}$ & $\begin{array}{l}4.92 \\
1.56\end{array}$ & $\begin{array}{l}6.17 \\
0.72\end{array}$ & 100 & - & - & - & - & - & Mountain & 17 \\
\hline \multirow[t]{2}{*}{ Hillside } & B & $\begin{array}{l}6.08 \\
1.24\end{array}$ & $\begin{array}{l}5.50 \\
1.83\end{array}$ & $\begin{array}{l}4.67 \\
1.23\end{array}$ & $\begin{array}{l}5.17 \\
1.90\end{array}$ & 25 & - & 17 & - & 50 & - & $\begin{array}{l}\text { Grass } \\
\text { Mountains }\end{array}$ & $\begin{array}{l}17 \\
17\end{array}$ \\
\hline & $\mathrm{S}$ & $\begin{array}{l}5.17 \\
1.53\end{array}$ & $\begin{array}{l}5.67 \\
1.37\end{array}$ & $\begin{array}{l}5.00 \\
1.21\end{array}$ & $\begin{array}{l}5.83 \\
1.27\end{array}$ & 92 & - & - & - & 08 & - & $\begin{array}{l}\text { Valley } \\
\text { Mountains }\end{array}$ & $\begin{array}{l}25 \\
17\end{array}$ \\
\hline \multirow[t]{2}{*}{$\mathrm{Hiss}$} & B & $\begin{array}{l}6.42 \\
1.00\end{array}$ & $\begin{array}{l}6.17 \\
1.03\end{array}$ & $\begin{array}{l}4.83 \\
1.53\end{array}$ & $\begin{array}{l}6.33 \\
0.89\end{array}$ & - & 100 & - & - & - & - & $\begin{array}{l}\text { Snake } \\
\text { Cat }\end{array}$ & $\begin{array}{l}42 \\
33\end{array}$ \\
\hline & $\mathrm{S}$ & $\begin{array}{l}5.50 \\
1.24\end{array}$ & $\begin{array}{l}4.92 \\
1.38\end{array}$ & $\begin{array}{l}4.50 \\
1.68\end{array}$ & $\begin{array}{l}5.42 \\
1.16\end{array}$ & 17 & 83 & - & - & - & - & $\begin{array}{l}\text { Snake } \\
\text { Cat }\end{array}$ & $\begin{array}{l}75 \\
17\end{array}$ \\
\hline \multirow[t]{2}{*}{ Honk } & B & $\begin{array}{l}6.00 \\
1.54\end{array}$ & $\begin{array}{l}5.92 \\
1.78\end{array}$ & $\begin{array}{l}4.17 \\
2.21\end{array}$ & $\begin{array}{l}5.00 \\
2.34\end{array}$ & - & 75 & - & - & - & - & Horn & 58 \\
\hline & $\mathrm{S}$ & $\begin{array}{l}5.33 \\
1.67\end{array}$ & $\begin{array}{l}5.00 \\
1.41\end{array}$ & $\begin{array}{l}4.50 \\
1.57\end{array}$ & $\begin{array}{l}5.58 \\
1.24\end{array}$ & 17 & 83 & - & - & - & - & Horn & 50 \\
\hline
\end{tabular}


APPENDIX (Continued)

\begin{tabular}{|c|c|c|c|c|c|c|c|c|c|c|c|c|c|}
\hline \multirow[b]{2}{*}{ Noun } & \multirow{2}{*}{$\begin{array}{l}\text { Subject } \\
\text { Group }\end{array}$} & \multirow[b]{2}{*}{ f } & \multirow[b]{2}{*}{ c } & \multirow[b]{2}{*}{$\mathrm{m}$} & \multirow[b]{2}{*}{$\mathrm{i}$} & \multicolumn{6}{|c|}{ Modality (\%) } & \multirow{2}{*}{$\begin{array}{c}\text { Strongest } \\
\text { Associates }\end{array}$} & \multirow[b]{2}{*}{$\%$} \\
\hline & & & & & & $(\mathrm{v}$ & $\mathbf{a}$ & tc & ts & $\mathbf{s p}$ & $\mathrm{sm})$ & & \\
\hline \multirow[t]{2}{*}{ Honor } & B & $\begin{array}{l}6.50 \\
0.67\end{array}$ & $\begin{array}{l}3.00 \\
2.00\end{array}$ & $\begin{array}{l}4.17 \\
1.85\end{array}$ & $\begin{array}{l}2.33 \\
2.27\end{array}$ & - & 08 & - & - & 17 & - & & \\
\hline & $\mathrm{s}$ & $\begin{array}{l}5.42 \\
1.56\end{array}$ & $\begin{array}{l}2.17 \\
0.83\end{array}$ & $\begin{array}{l}5.08 \\
1.56\end{array}$ & $\begin{array}{l}2.58 \\
2.39\end{array}$ & 33 & - & - & - & - & - & Glory & 33 \\
\hline \multirow[t]{2}{*}{ Hound } & B & $\begin{array}{l}6.67 \\
0.49\end{array}$ & $\begin{array}{l}5.08 \\
1.98\end{array}$ & $\begin{array}{l}4.33 \\
2.06\end{array}$ & $\begin{array}{l}4.75 \\
2.45\end{array}$ & 08 & 25 & 25 & - & 08 & 08 & Dog & 75 \\
\hline & $\mathrm{S}$ & $\begin{array}{l}5.25 \\
1.96\end{array}$ & $\begin{array}{l}4.58 \\
1.88\end{array}$ & $\begin{array}{l}4.67 \\
1.92\end{array}$ & $\begin{array}{l}4.92 \\
2.15\end{array}$ & 67 & 08 & 08 & - & - & - & Dog & 58 \\
\hline \multirow[t]{2}{*}{ Icebox } & $\mathbf{B}$ & $\begin{array}{l}6.58 \\
0.51\end{array}$ & $\begin{array}{l}6.17 \\
1.34\end{array}$ & $\begin{array}{l}5.00 \\
1.54\end{array}$ & $\begin{array}{l}6.33 \\
0.98\end{array}$ & 08 & 17 & 67 & - & 08 & - & \multicolumn{2}{|c|}{ Refrigerator 92} \\
\hline & $\mathrm{S}$ & $\begin{array}{l}5.08 \\
1.83\end{array}$ & $\begin{array}{l}6.00 \\
0.85\end{array}$ & $\begin{array}{l}4.58 \\
1.62\end{array}$ & $\begin{array}{l}5.67 \\
1.07\end{array}$ & 58 & - & 33 & - & 08 & - & $\begin{array}{l}\text { Cold } \\
\text { Freezer }\end{array}$ & $\begin{array}{l}33 \\
25\end{array}$ \\
\hline \multirow[t]{2}{*}{ Ignorance } & $\mathrm{B}$ & $\begin{array}{l}6.33 \\
0.78\end{array}$ & $\begin{array}{l}2.58 \\
2.02\end{array}$ & $\begin{array}{l}4.08 \\
2.11\end{array}$ & $\begin{array}{l}2.33 \\
2.42\end{array}$ & - & 17 & - & - & - & - & $\begin{array}{l}\text { Dumb } \\
\text { Bliss }\end{array}$ & $\begin{array}{l}42 \\
25\end{array}$ \\
\hline & $\mathrm{S}$ & $\begin{array}{l}5.25 \\
1.60\end{array}$ & $\begin{array}{l}2.25 \\
1.66\end{array}$ & $\begin{array}{l}4.83 \\
1.53\end{array}$ & $\begin{array}{l}2.08 \\
1.88\end{array}$ & 33 & - & - & - & - & - & $\begin{array}{l}\text { Stupidity } \\
\text { Bliss }\end{array}$ & $\begin{array}{l}50 \\
25\end{array}$ \\
\hline Intellect & $\mathrm{B}$ & $\begin{array}{l}6.58 \\
0.67\end{array}$ & $\begin{array}{l}2.33 \\
1.61\end{array}$ & $\begin{array}{l}4.00 \\
1.81\end{array}$ & $\begin{array}{l}1.83 \\
1.75\end{array}$ & - & - & - & - & 25 & - & \multicolumn{2}{|c|}{ Inte 11 igence 42} \\
\hline & $\mathrm{S}$ & $\begin{array}{l}5.08 \\
1.38\end{array}$ & $\begin{array}{l}2.17 \\
1.19\end{array}$ & $\begin{array}{l}5.58 \\
1.44\end{array}$ & $\begin{array}{l}1.58 \\
1.16\end{array}$ & 17 & 08 & - & - & - & - & $\begin{array}{l}\text { Smart } \\
\text { Inte } 11 \text { igenc } \\
\text { Rnowledge }\end{array}$ & $\begin{array}{l}25 \\
c e 17 \\
17\end{array}$ \\
\hline \multirow[t]{2}{*}{ Joy } & B & $\begin{array}{l}6.83 \\
0.39\end{array}$ & $\begin{array}{l}3.08 \\
2.11\end{array}$ & $\begin{array}{l}4.58 \\
1.62\end{array}$ & $\begin{array}{l}3.50 \\
2.84\end{array}$ & - & 25 & 08 & - & 08 & - & $\begin{array}{l}\text { Happy } \\
\text { Sorrow }\end{array}$ & $\begin{array}{l}58 \\
17\end{array}$ \\
\hline & $S$ & $\begin{array}{l}5.92 \\
1.38\end{array}$ & $\begin{array}{l}3.25 \\
1.82\end{array}$ & $\begin{array}{l}5.50 \\
1.38\end{array}$ & $\begin{array}{l}3.33 \\
2.31\end{array}$ & 42 & 08 & 08 & - & 08 & - & $\begin{array}{l}\text { Happy } \\
\text { Laughter }\end{array}$ & $\begin{array}{l}58 \\
17\end{array}$ \\
\hline \multirow[t]{2}{*}{ Juggler } & B & $\begin{array}{l}6.00 \\
1.13\end{array}$ & $\begin{array}{l}5.00 \\
1.86\end{array}$ & $\begin{array}{l}4.25 \\
1.82\end{array}$ & $\begin{array}{l}4.50 \\
2.67\end{array}$ & 25 & 08 & 33 & - & 17 & - & $\begin{array}{l}\text { Circus } \\
\text { Balls }\end{array}$ & $\begin{array}{l}33 \\
25\end{array}$ \\
\hline & $\mathrm{S}$ & $\begin{array}{l}5.17 \\
1.64\end{array}$ & $\begin{array}{l}5.25 \\
0.87\end{array}$ & $\begin{array}{l}4.08 \\
1.08\end{array}$ & $\begin{array}{l}5.75 \\
0.87\end{array}$ & 100 & $0-$ & - & - & - & - & $\begin{array}{l}\text { Circus } \\
\text { Clown }\end{array}$ & $\begin{array}{l}33 \\
17\end{array}$ \\
\hline \multirow[t]{2}{*}{ Jury } & B & $\begin{array}{l}6.42 \\
0.80\end{array}$ & $\begin{array}{l}5.00 \\
2.41\end{array}$ & $\begin{array}{l}4.92 \\
1.62\end{array}$ & $\begin{array}{l}4.25 \\
2.67\end{array}$ & 17 & 33 & - & - & 17 & - & $\begin{array}{l}\text { Trial } \\
\text { Court/Room }\end{array}$ & $\begin{array}{l}42 \\
33\end{array}$ \\
\hline & $\mathrm{S}$ & $\begin{array}{l}5.50 \\
1.38\end{array}$ & $\begin{array}{l}5.50 \\
1.17\end{array}$ & $\begin{array}{l}4.92 \\
1.51\end{array}$ & $\begin{array}{l}5.50 \\
0.80\end{array}$ & 100 & $0-$ & - & - & - & - & $\begin{array}{l}\text { Duty } \\
\text { Trial }\end{array}$ & $\begin{array}{l}25 \\
25\end{array}$ \\
\hline
\end{tabular}




\section{APPENDIX (Continued)}

\begin{tabular}{|c|c|c|c|c|c|c|c|c|c|c|c|c|c|}
\hline \multirow{2}{*}{ Noun } & \multirow{2}{*}{$\begin{array}{l}\text { Subject } \\
\text { Group }\end{array}$} & \multirow[b]{2}{*}{$\mathrm{f}$} & \multirow[b]{2}{*}{$\mathrm{c}$} & \multirow[b]{2}{*}{$\mathrm{m}$} & \multirow[b]{2}{*}{ i } & \multicolumn{6}{|c|}{ Modality (\%) } & \multirow{2}{*}{$\begin{array}{c}\text { Strongest } \\
\text { Associates }\end{array}$} & \multirow[b]{2}{*}{$\%$} \\
\hline & & & & & & $(\mathrm{v}$ & a & tc & ts & $\mathrm{sp}$ & $\mathrm{sm})$ & & \\
\hline \multirow[t]{4}{*}{ Kerchief } & B & 5.50 & 5.33 & 4.00 & 5.17 & \multirow[t]{2}{*}{17} & \multirow[t]{2}{*}{ - } & \multirow[t]{2}{*}{83} & \multirow[t]{2}{*}{-} & \multirow[t]{2}{*}{-} & \multirow[t]{2}{*}{-} & \multicolumn{2}{|c|}{ Handkerchief 42} \\
\hline & & 1.57 & 1.72 & 1.54 & 1.40 & & & & & & & Scarf & 25 \\
\hline & $S$ & 3.67 & 4.92 & 3.00 & 4.75 & 75 & - & 08 & - & - & - & Handkerchi & $f 25$ \\
\hline & & 1.83 & 1.98 & 1.65 & 2.14 & & & & & & & Hat & 17 \\
\hline \multirow[t]{3}{*}{ King } & B & 6.58 & 5.25 & 5.00 & 3.67 & \multirow[t]{2}{*}{17} & \multirow[t]{2}{*}{17} & \multirow[t]{2}{*}{08} & \multirow[t]{2}{*}{ - } & \multirow[t]{2}{*}{17} & \multirow[t]{2}{*}{-} & Queen & 50 \\
\hline & & 0.90 & 1.91 & 1.54 & 2.71 & & & & & & & Throne & 17 \\
\hline & S & $\begin{array}{l}5.58 \\
1.38\end{array}$ & $\begin{array}{l}5.33 \\
1.44\end{array}$ & $\begin{array}{l}5.25 \\
1.54\end{array}$ & $\begin{array}{l}5.67 \\
1.07\end{array}$ & 100 & - & - & - & - & - & Queen & 83 \\
\hline Knowledge & B & 6.58 & 2.67 & 4.58 & 2.42 & - & 08 & - & - & 17 & - & Informatio & $n 17$ \\
\hline & $\mathrm{S}$ & 5.67 & 2.58 & 5.42 & 1.75 & 17 & - & - & - & - & - & Intelligen & ce 33 \\
\hline & & 1.15 & 1.08 & 1.62 & 1.86 & & & & & & & Smart & 17 \\
\hline Landscape & B & 6.00 & 5.58 & 4.17 & 5.17 & 17 & - & 17 & - & 50 & 08 & Trees & 17 \\
\hline & & & & & & & & & & & & & \\
\hline & $\mathrm{S}$ & 5.08 & 5.42 & 5.17 & 5.67 & 92 & - & - & - & 08 & - & Drawing/ & \\
\hline & & 1.13 & 1.56 & 1.19 & & & & & & & & $\begin{array}{l}\text { Painting } \\
\text { Garden }\end{array}$ & $\begin{array}{l}17 \\
17\end{array}$ \\
\hline Laugh & B & 6.83 & 5.67 & 5.67 & 6.08 & 08 & 92 & - & - & - & - & Funny & 25 \\
\hline & & 0.39 & 1.50 & 1.07 & 1.08 & & & & & & & Cry & 17 \\
\hline & & & & & & & & & & & & & 17 \\
\hline & $S$ & $\begin{array}{l}6.33 \\
0.78\end{array}$ & $\begin{array}{l}5.67 \\
1.07\end{array}$ & $\begin{array}{l}5.50 \\
1.31\end{array}$ & $\begin{array}{l}5.83 \\
1.11\end{array}$ & 08 & 83 & - & - & 08 & - & Cry & 42 \\
\hline Laughter & B & 6.75 & 6.33 & 5.75 & 5.92 & - & 100 & - & - & - & - & Happiness & 33 \\
\hline & S & 6.25 & 5.33 & 5.17 & 5.83 & 08 & 83 & 08 & - & - & - & Happiness & 25 \\
\hline & & 0.87 & 1.16 & 1.40 & 1.03 & & & & & & & Tears & 17 \\
\hline Lemon & B & 6.67 & 6.58 & 5.42 & 6.58 & - & - & 25 & 58 & - & 17 & Fruit & 50 \\
\hline & & 0.65 & 0.67 & 1.44 & 0.67 & & & & & & & Pee1 & 17 \\
\hline & S & 5.67 & 6.17 & 5.00 & 5.92 & 50 & - & - & 50 & - & - & Peel & 33 \\
\hline & & 1.37 & 0.94 & 1.60 & 1.00 & & & & & & & Car & 25 \\
\hline Love & B & 7.00 & 3.33 & 5.50 & 3.67 & - & 08 & 25 & - & 08 & - & Hate & 25 \\
\hline & & 0.00 & 2.31 & 1.38 & 2.81 & & & & & & & Affection & 17 \\
\hline & S & 6.58 & 4.08 & 5.92 & 2.75 & 17 & - & 17 & - & 17 & - & Hate & 50 \\
\hline & & 0.67 & 2.02 & 1.08 & 2.14 & & & & & & & Cherish & 17 \\
\hline
\end{tabular}


APPENDIX (Continued)

\begin{tabular}{|c|c|c|c|c|c|c|c|c|c|c|c|c|c|}
\hline \multirow[b]{2}{*}{ Noun } & \multirow{2}{*}{$\begin{array}{l}\text { Subject } \\
\text { Group }\end{array}$} & \multirow[b]{2}{*}{ f } & \multirow[b]{2}{*}{ c } & \multirow[b]{2}{*}{$\mathrm{m}$} & \multirow[b]{2}{*}{$\mathrm{i}$} & \multicolumn{6}{|c|}{ Modality (\%) } & \multirow{2}{*}{$\begin{array}{c}\text { Strongest } \\
\text { Associates }\end{array}$} & \multirow[b]{2}{*}{$\%$} \\
\hline & & & & & & $(\mathrm{v}$ & a & tc & ts & $\mathrm{sp}$ & sm) & & \\
\hline \multirow[t]{3}{*}{ Maiden } & B & $\begin{array}{l}6.25 \\
1.06\end{array}$ & $\begin{array}{l}4.92 \\
2.15\end{array}$ & $\begin{array}{l}3.75 \\
1.82\end{array}$ & $\begin{array}{l}3.50 \\
2.54\end{array}$ & 170 & 08 & 25 & - & 08 & - & $\begin{array}{l}\text { Single/ } \\
\text { Unmarried }\end{array}$ & 25 \\
\hline & & & & & & & & & & & & Voyage & 25 \\
\hline & $\mathrm{s}$ & $\begin{array}{l}4.33 \\
2.02\end{array}$ & $\begin{array}{l}5.17 \\
1.53\end{array}$ & $\begin{array}{l}3.83 \\
1.12\end{array}$ & $\begin{array}{l}5.17 \\
1.64\end{array}$ & $92-$ & - & 08 & - & - & - & Fair & 17 \\
\hline \multirow[t]{2}{*}{ Mast } & B & $\begin{array}{l}4.83 \\
1.95\end{array}$ & $\begin{array}{l}5.08 \\
2.07\end{array}$ & $\begin{array}{l}3.92 \\
1.78\end{array}$ & $\begin{array}{l}4.58 \\
1.83\end{array}$ & $17-$ & - & 42 & - & 33 & - & $\begin{array}{l}\text { Ship } \\
\text { Sail }\end{array}$ & $\begin{array}{l}33 \\
25\end{array}$ \\
\hline & $\mathrm{s}$ & $\begin{array}{l}5.00 \\
1.95\end{array}$ & $\begin{array}{l}5.58 \\
1.24\end{array}$ & $\begin{array}{l}4.17 \\
1.80\end{array}$ & $\begin{array}{l}5.25 \\
1.42\end{array}$ & $92-$ & - & 08 & - & - & - & $\begin{array}{l}\text { Ship } \\
\text { Boat }\end{array}$ & $\begin{array}{l}42 \\
25\end{array}$ \\
\hline \multirow[t]{2}{*}{ Meadow } & B & $\begin{array}{l}6.25 \\
1.14\end{array}$ & $\begin{array}{l}5.50 \\
1.68\end{array}$ & $\begin{array}{l}4.58 \\
1.51\end{array}$ & $\begin{array}{l}5.67 \\
1.37\end{array}$ & $25-$ & - & 17 & - & 50 & 08 & Green & 17 \\
\hline & $\mathrm{s}$ & $\begin{array}{l}4.75 \\
1.75\end{array}$ & $\begin{array}{l}5.08 \\
1.44\end{array}$ & $\begin{array}{l}5.42 \\
1.38\end{array}$ & $\begin{array}{l}5.75 \\
1.34\end{array}$ & 100 & - & - & - & - & - & $\begin{array}{l}\text { Field } \\
\text { Prairie }\end{array}$ & $\begin{array}{l}17 \\
17\end{array}$ \\
\hline \multirow[t]{2}{*}{ Memory } & B & $\begin{array}{l}6.92 \\
0.29\end{array}$ & $\begin{array}{l}3.58 \\
2.50\end{array}$ & $\begin{array}{l}4.92 \\
1.73\end{array}$ & $\begin{array}{l}2.75 \\
2.53\end{array}$ & -1 & 17 & - & - & 17 & - & $\begin{array}{l}\text { Remember/ } \\
\text { Recall } \\
\text { Brain }\end{array}$ & $\begin{array}{l}25 \\
17\end{array}$ \\
\hline & s & $\begin{array}{l}5.58 \\
1.31\end{array}$ & $\begin{array}{l}2.75 \\
1.71\end{array}$ & $\begin{array}{l}5.00 \\
1.28\end{array}$ & $\begin{array}{l}2.50 \\
2.32\end{array}$ & 17 & - & - & - & 25 & - & - & \\
\hline \multirow[t]{2}{*}{ Mirror } & B & $\begin{array}{l}5.92 \\
1.56\end{array}$ & $\begin{array}{l}4.92 \\
2.43\end{array}$ & $\begin{array}{l}4.42 \\
1.93\end{array}$ & $\begin{array}{l}5.08 \\
2.02\end{array}$ & 25 & - & 42 & - & 25 & - & $\begin{array}{l}\text { Glass } \\
\text { Image }\end{array}$ & $\begin{array}{l}42 \\
17\end{array}$ \\
\hline & s & $\begin{array}{l}6.08 \\
1.16\end{array}$ & $\begin{array}{l}6.17 \\
0.94\end{array}$ & $\begin{array}{l}4.42 \\
1.62\end{array}$ & $\begin{array}{l}6.25 \\
0.97\end{array}$ & 100 & - & - & - & - & - & Reflection & 33 \\
\hline \multirow[t]{2}{*}{ Misconceptio } & $n$ B & $\begin{array}{l}6.33 \\
0.89\end{array}$ & $\begin{array}{l}2.50 \\
1.83\end{array}$ & $\begin{array}{l}3.58 \\
1.83\end{array}$ & $\begin{array}{l}2.08 \\
2.07\end{array}$ & - & 08 & - & - & 08 & - & $\begin{array}{l}\text { Misunder- } \\
\text { standing }\end{array}$ & 33 \\
\hline & $\mathrm{s}$ & $\begin{array}{l}4.42 \\
1.93\end{array}$ & $\begin{array}{l}2.08 \\
1.08\end{array}$ & $\begin{array}{l}4.08 \\
1.88\end{array}$ & $\begin{array}{l}1.42 \\
1.16\end{array}$ & - & - & - & - & 08 & - & Wrong & 33 \\
\hline \multirow[t]{2}{*}{ Monk } & B & $\begin{array}{l}6.42 \\
1.00\end{array}$ & $\begin{array}{l}5.00 \\
2.00\end{array}$ & $\begin{array}{l}3.50 \\
1.51\end{array}$ & $\begin{array}{l}4.25 \\
2.45\end{array}$ & 17 & 17 & 17 & - & 25 & - & $\begin{array}{l}\text { Monastery } \\
\text { Priest }\end{array}$ & $\begin{array}{l}42 \\
17\end{array}$ \\
\hline & $\mathrm{s}$ & $\begin{array}{l}4.58 \\
1.78\end{array}$ & $\begin{array}{l}5.75 \\
1.06\end{array}$ & $\begin{array}{l}4.33 \\
1.37\end{array}$ & $\begin{array}{l}5.83 \\
0.94\end{array}$ & 100 & - & - & - & - & - & $\begin{array}{l}\text { Priest } \\
\text { Monastery }\end{array}$ & $\begin{array}{l}33 \\
17\end{array}$ \\
\hline \multirow[t]{2}{*}{ Mountain } & B & $\begin{array}{l}6.33 \\
1.15\end{array}$ & $\begin{array}{l}5.83 \\
1.40\end{array}$ & $\begin{array}{l}5.00 \\
1.28\end{array}$ & $\begin{array}{l}5.67 \\
1.44\end{array}$ & 17 & - & 25 & - & 58 & - & High & 25 \\
\hline & $\mathrm{s}$ & $\begin{array}{l}5.75 \\
1.36\end{array}$ & $\begin{array}{l}6.25 \\
0.87\end{array}$ & $\begin{array}{l}5.08 \\
1.51\end{array}$ & $\begin{array}{l}6.33 \\
0.65\end{array}$ & 100 & - & - & - & - & - & Peak & 25 \\
\hline
\end{tabular}


APPENDIX (Continued)

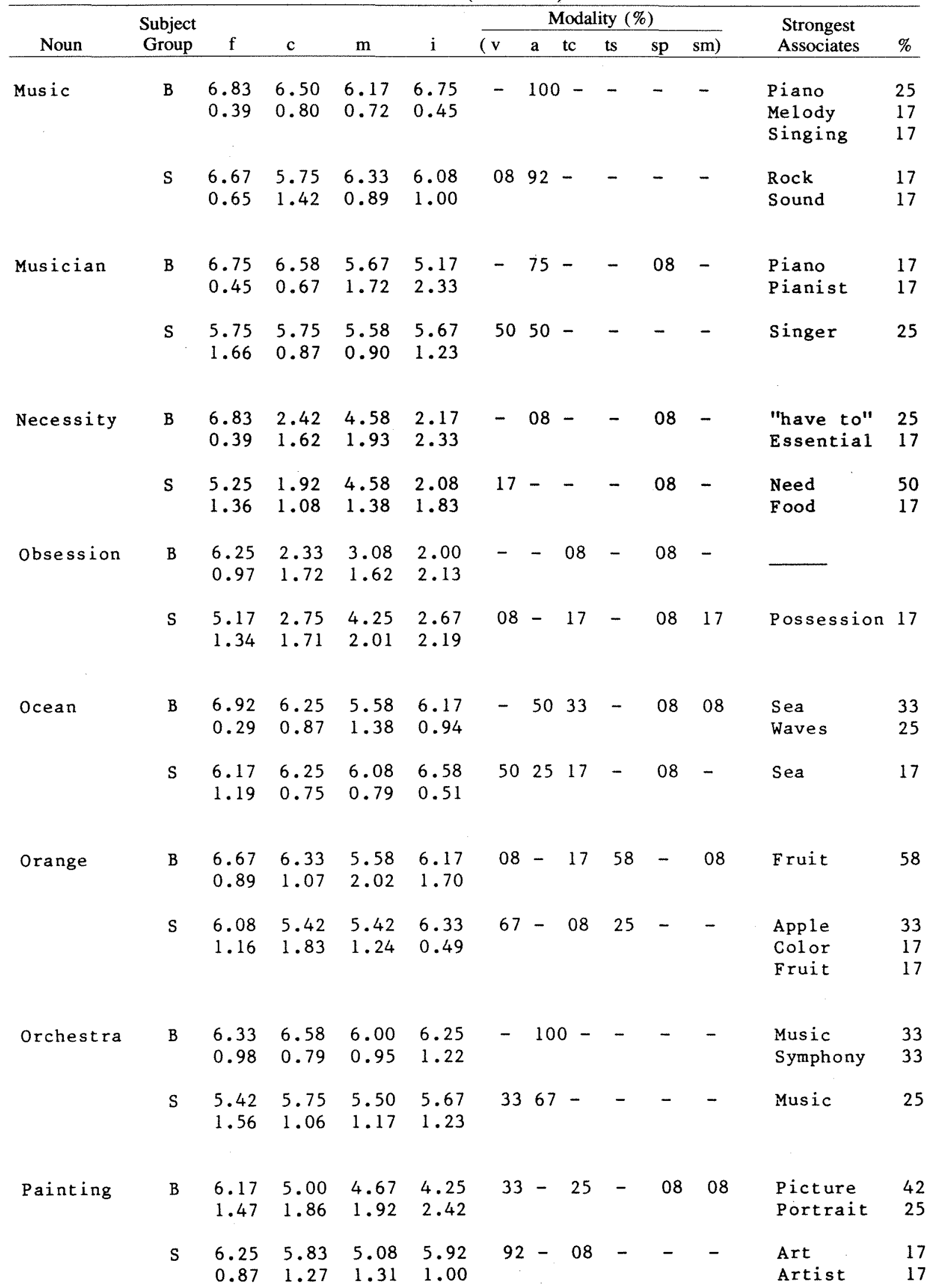




\section{APPENDIX (Continued)}

\begin{tabular}{|c|c|c|c|c|c|c|c|c|c|c|c|c|c|}
\hline \multirow{2}{*}{ Noun } & \multirow{2}{*}{$\begin{array}{c}\text { Subject } \\
\text { Group }\end{array}$} & \multirow[b]{2}{*}{$\mathrm{f}$} & \multirow[b]{2}{*}{$\mathrm{c}$} & \multirow[b]{2}{*}{$\mathrm{m}$} & \multirow[b]{2}{*}{$\mathrm{i}$} & \multicolumn{6}{|c|}{ Modality (\%) } & \multirow{2}{*}{$\begin{array}{c}\text { Strongest } \\
\text { Associates }\end{array}$} & \multirow[b]{2}{*}{$\%$} \\
\hline & & & & & & $(\mathrm{v}$ & a & tc & ts & $\mathrm{sp}$ & sm) & & \\
\hline \multirow[t]{3}{*}{ Palace } & B & 5.83 & 5.08 & 4.33 & 5.33 & 17 & - & 33 & - & 50 & - & King & 25 \\
\hline & & & & & & & & & & & & $\begin{array}{l}\text { Castle } \\
\text { Mansion }\end{array}$ & $\begin{array}{l}17 \\
17\end{array}$ \\
\hline & $\mathrm{s}$ & $\begin{array}{l}5.25 \\
1.86\end{array}$ & $\begin{array}{l}5.75 \\
1.22\end{array}$ & $\begin{array}{l}5.00 \\
1.76\end{array}$ & $\begin{array}{l}5.58 \\
1.38\end{array}$ & 100 & $0-$ & - & - & - & - & Castle & 33 \\
\hline \multirow[t]{4}{*}{ Pencil } & B & 6.50 & 5.58 & 5.08 & 6.25 & 08 & 08 & 83 & - & - & - & Writing & 58 \\
\hline & & & & & & & & & & & & & \\
\hline & $\mathrm{s}$ & 6.42 & 6.50 & 5.67 & 6.42 & 67 & - & 33 & - & - & - & Pen & 42 \\
\hline & & & & & & & & & & & & $\begin{array}{l}\text { Writing } \\
\text { Erasure }\end{array}$ & $\begin{array}{l}17 \\
17\end{array}$ \\
\hline \multirow[t]{4}{*}{ Pop } & B & 6.75 & 6.50 & 5.50 & 6.58 & - & 75 & - & - & - & 08 & Corn & 42 \\
\hline & & 0.45 & 0.90 & 1.24 & 0.90 & & & & & & & Sound & 17 \\
\hline & $\mathrm{s}$ & 5.33 & 4.83 & 4.17 & 5.33 & 08 & 75 & - & 08 & - & - & Wease 1 & 25 \\
\hline & & 1.61 & 1.53 & 1.75 & 1.72 & & & & & & & Soda & 17 \\
\hline \multirow[t]{4}{*}{ Portrait } & B & 6.08 & 4.83 & 4.42 & 4.67 & 25 & - & 33 & - & 17 & - & Picture & 42 \\
\hline & & 1.44 & 2.44 & 2.02 & 2.35 & & & & & & & Painting & 17 \\
\hline & $\mathrm{s}$ & 5.08 & 5.67 & 5.33 & 6.17 & 100 & 0 - & - & - & - & - & Painting & 33 \\
\hline & & 1.62 & 1.15 & 1.44 & 0.83 & & & & & & & Lady & 17 \\
\hline \multirow[t]{4}{*}{ Poster } & B & 6.17 & 5.08 & 4.17 & 4.67 & 33 & - & 42 & - & 08 & - & Picture & 50 \\
\hline & & 1.19 & 2.19 & 2.12 & 2.10 & & & & & & & & \\
\hline & S & 5.42 & 5.92 & 4.67 & 6.00 & 100 & $0-$ & - & - & - & - & Board & 42 \\
\hline & & 1.68 & 0.67 & 1.50 & 1.21 & & & & & & & Picture & 17 \\
\hline \multirow[t]{4}{*}{ Potato } & B & 6.83 & 6.58 & 5.67 & 6.92 & - & - & 58 & 42 & - & - & Vegetable & 25 \\
\hline & & 0.35 & 0.01 & 1.30 & 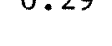 & & & & & & & & \\
\hline & $S$ & 5.83 & 6.42 & 4.67 & 6.33 & 67 & - & - & 33 & - & - & Head & 42 \\
\hline & & 1.27 & 0.67 & 1.44 & 0.78 & & & & & & & Fries & 17 \\
\hline \multirow[t]{3}{*}{ Prairie } & B & 6.00 & 5.33 & 4.25 & 4.83 & 17 & 17 & 17 & - & 42 & - & Dog & 25 \\
\hline & & 1.04 & 1.83 & 1.66 & 1.80 & & & & & & & Grass & 25 \\
\hline & s & $\begin{array}{l}4.83 \\
1.85\end{array}$ & $\begin{array}{l}5.25 \\
1.14\end{array}$ & $\begin{array}{l}5.33 \\
1.15\end{array}$ & $\begin{array}{l}5.42 \\
1.08\end{array}$ & 100 & $0-$ & - & - & - & - & Dog & 58 \\
\hline \multirow[t]{3}{*}{ Prestige } & B & 6.25 & 2.92 & 4.00 & 2.25 & - & - & 08 & - & 17 & - & Pride & 25 \\
\hline & & 0.87 & 1.78 & 1.86 & 2.14 & & & & & & & & \\
\hline & $\mathrm{s}$ & $\begin{array}{l}4.75 \\
1.60\end{array}$ & $\begin{array}{l}2.50 \\
1.09\end{array}$ & $\begin{array}{l}3.92 \\
1.38\end{array}$ & $\begin{array}{l}1.83 \\
1.53\end{array}$ & 17 & - & - & - & 08 & - & Honor & 42 \\
\hline
\end{tabular}


APPENDIX (Continued)

\begin{tabular}{|c|c|c|c|c|c|c|c|c|c|c|c|c|c|}
\hline \multirow[b]{2}{*}{ Noun } & \multirow{2}{*}{$\begin{array}{l}\text { Subject } \\
\text { Group }\end{array}$} & \multirow[b]{2}{*}{$\mathrm{f}$} & \multirow[b]{2}{*}{ c } & \multirow[b]{2}{*}{$\mathrm{m}$} & \multirow[b]{2}{*}{$\mathrm{i}$} & \multicolumn{6}{|c|}{ Modality (\%) } & \multirow{2}{*}{$\begin{array}{c}\text { Strongest } \\
\text { Associates }\end{array}$} & \multirow[b]{2}{*}{$\%$} \\
\hline & & & & & & (v & $\mathrm{a}$ & tc & ts & sp & sm) & & \\
\hline \multirow[t]{2}{*}{ Pride } & B & $\begin{array}{l}6.50 \\
0.90\end{array}$ & $\begin{array}{l}2.25 \\
1.60\end{array}$ & $\begin{array}{l}4.08 \\
2.11\end{array}$ & $\begin{array}{l}2.42 \\
2.57\end{array}$ & - & 08 & - & - & 08 & - & Proud & 25 \\
\hline & $\mathrm{s}$ & $\begin{array}{l}5.25 \\
1.71\end{array}$ & $\begin{array}{l}2.75 \\
1.66\end{array}$ & $\begin{array}{l}4.50 \\
1.88\end{array}$ & $\begin{array}{l}2.25 \\
2.05\end{array}$ & 08 & - & 08 & - & 17 & - & & \\
\hline \multirow[t]{2}{*}{ Professor } & B & $\begin{array}{l}6.50 \\
0.67\end{array}$ & $\begin{array}{l}5.50 \\
1.73\end{array}$ & $\begin{array}{l}5.42 \\
1.38\end{array}$ & $\begin{array}{l}4.92 \\
2.15\end{array}$ & 08 & 58 & 17 & - & - & - & $\begin{array}{l}\text { Teacher } \\
\text { College }\end{array}$ & $\begin{array}{l}42 \\
25\end{array}$ \\
\hline & $S$ & $\begin{array}{l}5.75 \\
1.66\end{array}$ & $\begin{array}{l}5.75 \\
1.22\end{array}$ & $\begin{array}{l}5.50 \\
1.31\end{array}$ & $\begin{array}{l}5.25 \\
1.14\end{array}$ & 83 & 17 & - & - & - & - & $\begin{array}{l}\text { Teacher } \\
\text { Instructor }\end{array}$ & $\begin{array}{l}42 \\
17\end{array}$ \\
\hline \multirow[t]{2}{*}{ Rail road } & B & $\begin{array}{l}6.42 \\
0.90\end{array}$ & $\begin{array}{l}5.83 \\
1.80\end{array}$ & $\begin{array}{l}5.25 \\
1.54\end{array}$ & $\begin{array}{l}5.67 \\
1.87\end{array}$ & 08 & 33 & 25 & - & 25 & - & Train & 67 \\
\hline & $\mathrm{s}$ & $\begin{array}{l}5.58 \\
1.44\end{array}$ & $\begin{array}{l}6.00 \\
0.95\end{array}$ & $\begin{array}{l}5.17 \\
1.47\end{array}$ & $\begin{array}{l}6.00 \\
0.85\end{array}$ & 83 & 17 & - & - & - & - & $\begin{array}{l}\text { Train } \\
\text { Car } \\
\text { Ties } \\
\text { Tracks }\end{array}$ & $\begin{array}{l}25 \\
17 \\
17 \\
17\end{array}$ \\
\hline \multirow[t]{2}{*}{ Rainbow } & B & $\begin{array}{l}5.92 \\
1.73\end{array}$ & $\begin{array}{l}3.92 \\
2.71\end{array}$ & $\begin{array}{l}3.67 \\
1.87\end{array}$ & $\begin{array}{l}3.17 \\
2.17\end{array}$ & 25 & - & - & - & 25 & - & $\begin{array}{l}\text { Colors } \\
\text { Beauty }\end{array}$ & $\begin{array}{l}50 \\
25\end{array}$ \\
\hline & $\mathrm{s}$ & $\begin{array}{l}6.00 \\
0.95\end{array}$ & $\begin{array}{l}5.67 \\
1.44\end{array}$ & $\begin{array}{l}4.83 \\
1.80\end{array}$ & $\begin{array}{l}6.08 \\
1.08\end{array}$ & 100 & - & - & - & - & - & Colors & 33 \\
\hline \multirow[t]{2}{*}{ Rattle } & B & $\begin{array}{l}6.58 \\
0.67\end{array}$ & $\begin{array}{l}6.42 \\
0.90\end{array}$ & $\begin{array}{l}5.58 \\
1.16\end{array}$ & $\begin{array}{l}6.33 \\
0.98\end{array}$ & - & 92 & 08 & - & - & - & $\begin{array}{l}\text { Noise/Soun } \\
\text { Snake }\end{array}$ & $\begin{array}{r}833 \\
33\end{array}$ \\
\hline & s & $\begin{array}{l}5.17 \\
1.80\end{array}$ & $\begin{array}{l}5.50 \\
1.24\end{array}$ & $\begin{array}{l}4.50 \\
1.78\end{array}$ & $\begin{array}{l}5.33 \\
1.15\end{array}$ & 17 & 83 & - & - & - & - & $\begin{array}{l}\text { Snake } \\
\text { Baby }\end{array}$ & $\begin{array}{l}67 \\
25\end{array}$ \\
\hline \multirow[t]{2}{*}{ Roar } & B & $\begin{array}{l}6.75 \\
0.45\end{array}$ & $\begin{array}{l}6.58 \\
0.90\end{array}$ & $\begin{array}{l}5.42 \\
1.08\end{array}$ & $\begin{array}{l}6.50 \\
0.80\end{array}$ & - & 100 & - & - & - & - & $\begin{array}{l}\text { Lion } \\
\text { Loud }\end{array}$ & $\begin{array}{l}33 \\
25\end{array}$ \\
\hline & $\mathrm{s}$ & $\begin{array}{l}5.25 \\
1.82\end{array}$ & $\begin{array}{l}5.50 \\
1.09\end{array}$ & $\begin{array}{l}4.33 \\
0.98\end{array}$ & $\begin{array}{l}5.50 \\
1.00\end{array}$ & - & 100 & - & - & - & - & Lion & 75 \\
\hline \multirow[t]{2}{*}{ Roof } & B & $\begin{array}{l}6.08 \\
1.83\end{array}$ & $\begin{array}{l}5.75 \\
1.76\end{array}$ & $\begin{array}{l}5.08 \\
1.24\end{array}$ & $\begin{array}{l}5.83 \\
1.19\end{array}$ & 25 & - & 42 & - & $33^{\prime}$ & - & $\begin{array}{l}\text { Top } \\
\text { House }\end{array}$ & $\begin{array}{l}42 \\
25\end{array}$ \\
\hline & S & $\begin{array}{l}5.92 \\
1.16\end{array}$ & $\begin{array}{l}5.58 \\
1.51\end{array}$ & $\begin{array}{l}4.67 \\
1.61\end{array}$ & $\begin{array}{l}5.42 \\
1.24\end{array}$ & 75 & -1 & 08 & - & 17 & - & $\begin{array}{l}\text { House } \\
\text { Head }\end{array}$ & $\begin{array}{l}33 \\
17\end{array}$ \\
\hline \multirow[t]{2}{*}{ Scarlet } & B & $\begin{array}{l}5.92 \\
1.73\end{array}$ & $\begin{array}{l}3.67 \\
2.35\end{array}$ & $\begin{array}{l}3.75 \\
1.82\end{array}$ & $\begin{array}{l}3.17 \\
2.29\end{array}$ & 33 & - & - & - & 08 & - & $\begin{array}{l}\text { Red } \\
\text { O'Hara }\end{array}$ & $\begin{array}{l}50 \\
33\end{array}$ \\
\hline & S & $\begin{array}{l}5.08 \\
1.93\end{array}$ & $\begin{array}{l}5.00 \\
2.13\end{array}$ & $\begin{array}{l}5.17 \\
1.64\end{array}$ & $\begin{array}{l}5.92 \\
1.00\end{array}$ & 100 & $0-$ & - & - & - & - & $\begin{array}{l}\text { O'Hara } \\
\text { Red }\end{array}$ & $\begin{array}{l}42 \\
17\end{array}$ \\
\hline
\end{tabular}


APPENDIX (Continued)

\begin{tabular}{|c|c|c|c|c|c|c|c|c|c|c|c|c|c|}
\hline \multirow[b]{2}{*}{ Noun } & \multirow{2}{*}{$\begin{array}{l}\text { Subject } \\
\text { Group }\end{array}$} & \multirow[b]{2}{*}{$\mathrm{f}$} & \multirow[b]{2}{*}{$\mathrm{c}$} & \multirow[b]{2}{*}{$\mathrm{m}$} & \multirow[b]{2}{*}{$\mathrm{i}$} & \multicolumn{6}{|c|}{ Modality (\%) } & \multirow{2}{*}{$\begin{array}{c}\text { Strongest } \\
\text { Associates } \\
\end{array}$} & \multirow[b]{2}{*}{$\%$} \\
\hline & & & & & & $(\mathrm{v}$ & a t & tc & ts & $\mathrm{sp}$ & $\mathrm{sm})$ & & \\
\hline \multirow[t]{2}{*}{ Scream } & B & $\begin{array}{l}6.75 \\
0.45\end{array}$ & $\begin{array}{l}6.67 \\
0.65\end{array}$ & $\begin{array}{l}5.00 \\
1.60\end{array}$ & $\begin{array}{l}6.08 \\
1.51\end{array}$ & - & 100 & - & - & - & - & $\begin{array}{l}\text { Yell } \\
\text { Loud }\end{array}$ & $\begin{array}{l}25 \\
17\end{array}$ \\
\hline & $\mathrm{s}$ & $\begin{array}{l}5.58 \\
1.24\end{array}$ & $\begin{array}{l}5.42 \\
1.31\end{array}$ & $\begin{array}{l}5.50 \\
0.90\end{array}$ & $\begin{array}{l}5.92 \\
1.00\end{array}$ & 08 & $92-$ & - & - & - & - & _ & \\
\hline \multirow[t]{2}{*}{ Sensation } & B & $\begin{array}{l}6.58 \\
0.67\end{array}$ & $\begin{array}{l}4.00 \\
2.00\end{array}$ & $\begin{array}{l}3.33 \\
1.72\end{array}$ & $\begin{array}{l}3.75 \\
2.70\end{array}$ & - & -6 & 67 & - & - & - & Feeling & 50 \\
\hline & s & $\begin{array}{l}5.83 \\
0.83\end{array}$ & $\begin{array}{l}3.42 \\
2.19\end{array}$ & $\begin{array}{l}4.58 \\
1.98\end{array}$ & $\begin{array}{l}2.75 \\
2.30\end{array}$ & - & -5 & 50 & - & - & - & Feeling & 67 \\
\hline \multirow[t]{2}{*}{ Sentiment } & B & $\begin{array}{l}5.92 \\
1.08\end{array}$ & $\begin{array}{l}2.33 \\
1.67\end{array}$ & $\begin{array}{l}2.92 \\
1.78\end{array}$ & $\begin{array}{l}2.42 \\
2.57\end{array}$ & - & -0 & 08 & - & 08 & - & Feeling & 58 \\
\hline & $S$ & $\begin{array}{l}4.58 \\
1.68\end{array}$ & $\begin{array}{l}2.58 \\
1.31\end{array}$ & $\begin{array}{l}3.83 \\
1.75\end{array}$ & $\begin{array}{l}2.00 \\
1.60\end{array}$ & 25 & -0 & 08 & - & 08 & - & Feeling & 17 \\
\hline \multirow[t]{2}{*}{ Sha dow } & B & $\begin{array}{l}6.17 \\
1.40\end{array}$ & $\begin{array}{l}4.58 \\
2.64\end{array}$ & $\begin{array}{l}3.58 \\
2.11\end{array}$ & $\begin{array}{l}3.83 \\
2.66\end{array}$ & 33 & $08-$ & - & - & 08 & - & Image & 25 \\
\hline & $S$ & $\begin{array}{l}5.50 \\
1.38\end{array}$ & $\begin{array}{l}4.75 \\
1.60\end{array}$ & $\begin{array}{l}4.58 \\
1.73\end{array}$ & $\begin{array}{l}5.25 \\
1.54\end{array}$ & 100 & - & - & - & - & - & Darkness & 17 \\
\hline \multirow[t]{2}{*}{ Shame } & B & $\begin{array}{l}6.42 \\
0.90\end{array}$ & $\begin{array}{l}2.58 \\
1.56\end{array}$ & $\begin{array}{l}3.83 \\
1.70\end{array}$ & $\begin{array}{l}2.42 \\
2.57\end{array}$ & - & 08 & - & - & 08 & - & $\begin{array}{l}\text { Embarrasse } \\
\text { Regret }\end{array}$ & $\begin{array}{r}d 7 \\
17\end{array}$ \\
\hline & $S$ & $\begin{array}{l}5.25 \\
1.36\end{array}$ & $\begin{array}{l}3.00 \\
1.60\end{array}$ & $\begin{array}{l}4.58 \\
1.93\end{array}$ & $\begin{array}{l}3.00 \\
2.45\end{array}$ & 17 & -1 & 17 & - & 17 & - & Greed & 17 \\
\hline \multirow[t]{2}{*}{ Shoes } & B & $\begin{array}{l}6.92 \\
0.29\end{array}$ & $\begin{array}{l}6.75 \\
0.87\end{array}$ & $\begin{array}{l}5.33 \\
1.56\end{array}$ & $\begin{array}{l}6.83 \\
0.39\end{array}$ & 08 & $-\varepsilon$ & 83 & - & 08 & - & $\begin{array}{l}\text { Feet } \\
\text { Socks }\end{array}$ & $\begin{array}{l}58 \\
25\end{array}$ \\
\hline & $\mathrm{s}$ & $\begin{array}{l}6.25 \\
1.14\end{array}$ & $\begin{array}{l}6.25 \\
1.22\end{array}$ & $\begin{array}{l}4.92 \\
1.68\end{array}$ & $\begin{array}{l}6.08 \\
1.16\end{array}$ & 100 & $0-$ & - & - & - & - & Feet & 33 \\
\hline \multirow[t]{2}{*}{ Shotgun } & B & $\begin{array}{l}6.17 \\
1.34\end{array}$ & $\begin{array}{l}5.92 \\
1.78\end{array}$ & $\begin{array}{l}5.25 \\
1.60\end{array}$ & $\begin{array}{l}6.00 \\
1.04\end{array}$ & - & 831 & 17 & - & - & - & $\begin{array}{l}\text { Weapon } \\
\text { Hunting }\end{array}$ & $\begin{array}{l}25 \\
17\end{array}$ \\
\hline & s & $\begin{array}{l}5.58 \\
1.56\end{array}$ & $\begin{array}{l}6.17 \\
1.03\end{array}$ & $\begin{array}{l}5.25 \\
1.06\end{array}$ & $\begin{array}{l}6.08 \\
0.80\end{array}$ & 67 & 33 & - & - & - & - & $\begin{array}{l}\text { Blast } \\
\text { Rifle }\end{array}$ & $\begin{array}{l}17 \\
17\end{array}$ \\
\hline \multirow[t]{2}{*}{ Shout } & B & $\begin{array}{l}6.67 \\
0.65\end{array}$ & $\begin{array}{l}6.50 \\
0.80\end{array}$ & $\begin{array}{l}5.42 \\
1.51\end{array}$ & $\begin{array}{l}6.67 \\
0.65\end{array}$ & - & 100 & - & - & - & - & $\begin{array}{l}\text { Loud } \\
\text { Yel1 }\end{array}$ & $\begin{array}{l}42 \\
33\end{array}$ \\
\hline & s & $\begin{array}{l}5.75 \\
1.22\end{array}$ & $\begin{array}{l}4.58 \\
1.78\end{array}$ & $\begin{array}{l}4.67 \\
1.83\end{array}$ & $\begin{array}{l}5.58 \\
1.31\end{array}$ & 08 & 92 & - & - & - & - & $\begin{array}{l}\text { Yell } \\
\text { Loud } \\
\text { Scream }\end{array}$ & $\begin{array}{l}50 \\
17 \\
17\end{array}$ \\
\hline
\end{tabular}




\begin{tabular}{|c|c|c|c|c|c|c|c|c|c|c|c|c|c|}
\hline \multirow[b]{2}{*}{ Noun } & \multirow{2}{*}{$\begin{array}{c}\text { Subject } \\
\text { Group }\end{array}$} & \multirow[b]{2}{*}{$\mathbf{f}$} & \multirow[b]{2}{*}{$\mathrm{c}$} & \multirow[b]{2}{*}{$\mathrm{m}$} & \multirow[b]{2}{*}{$\mathrm{i}$} & \multicolumn{6}{|c|}{ Modality (\%) } & \multirow{2}{*}{$\begin{array}{l}\text { Strongest } \\
\text { Associates }\end{array}$} & \multirow[b]{2}{*}{$\%$} \\
\hline & & & & & & $(\mathrm{v}$ & $\mathrm{a}$ & tc & ts & sp & $\mathrm{sm})$ & & \\
\hline \multirow[t]{2}{*}{ Singer } & B & $\begin{array}{l}6.83 \\
0.39\end{array}$ & $\begin{array}{l}6.50 \\
0.90\end{array}$ & $\begin{array}{l}6.25 \\
0.62\end{array}$ & $\begin{array}{l}6.17 \\
1.80\end{array}$ & - & 83 & 08 & - & - & - & Music & 33 \\
\hline & $\mathrm{S}$ & $\begin{array}{l}6.00 \\
1.13\end{array}$ & $\begin{array}{l}5.58 \\
1.16\end{array}$ & $\begin{array}{l}5.42 \\
1.38\end{array}$ & $\begin{array}{l}5.67 \\
1.15\end{array}$ & 58 & 42 & - & - & - & - & $\begin{array}{l}\text { Dancer } \\
\text { Microphone }\end{array}$ & $\begin{array}{l}25 \\
17\end{array}$ \\
\hline \multirow[t]{2}{*}{ Situation } & B & $\begin{array}{l}6.42 \\
0.79\end{array}$ & $\begin{array}{l}3.00 \\
2.09\end{array}$ & $\begin{array}{l}4.00 \\
1.60\end{array}$ & $\begin{array}{l}2.25 \\
2.14\end{array}$ & - & 08 & - & - & 17 & - & \multicolumn{2}{|c|}{$\begin{array}{l}\text { Circumstance } 17 \\
\text { Predicament } 17\end{array}$} \\
\hline & $\mathrm{S}$ & $\begin{array}{l}5.83 \\
0.94\end{array}$ & $\begin{array}{l}2.33 \\
1.56\end{array}$ & $\begin{array}{l}3.42 \\
1.44\end{array}$ & $\begin{array}{l}1.42 \\
1.16\end{array}$ & - & - & - & - & 08 & - & Comedy & 33 \\
\hline \multirow[t]{2}{*}{ Skillet } & B & $\begin{array}{l}6.67 \\
0.65\end{array}$ & $\begin{array}{l}6.67 \\
0.49\end{array}$ & $\begin{array}{l}5.75 \\
1.71\end{array}$ & $\begin{array}{l}6.58 \\
0.67\end{array}$ & - & - & 100 & - & - & - & $\begin{array}{l}\text { Cook } \\
\text { Cooking }\end{array}$ & $\begin{array}{l}17 \\
17\end{array}$ \\
\hline & $S$ & $\begin{array}{l}5.33 \\
1.23\end{array}$ & $\begin{array}{l}6.08 \\
1.08\end{array}$ & $\begin{array}{l}4.42 \\
1.73\end{array}$ & $\begin{array}{l}6.33 \\
0.65\end{array}$ & 75 & 170 & 08 & - & - & - & $\begin{array}{l}\text { Pan } \\
\text { Eggs }\end{array}$ & $\begin{array}{l}33 \\
25\end{array}$ \\
\hline \multirow[t]{2}{*}{ Sky } & B & $\begin{array}{l}6.25 \\
1.71\end{array}$ & $\begin{array}{l}4.67 \\
2.35\end{array}$ & $\begin{array}{l}4.17 \\
2.08\end{array}$ & $\begin{array}{l}4.75 \\
1.86\end{array}$ & 25 & - & 08 & - & 50 & - & Blue & 50 \\
\hline & S & $\begin{array}{l}6.25 \\
0.97\end{array}$ & $\begin{array}{l}5.33 \\
1.44\end{array}$ & $\begin{array}{l}4.92 \\
1.51\end{array}$ & $\begin{array}{l}6.25 \\
0.75\end{array}$ & 100 & $0-$ & - & - & - & - & Blue & 58 \\
\hline \multirow[t]{2}{*}{ Snake } & B & $\begin{array}{l}6.33 \\
0.98\end{array}$ & $\begin{array}{l}5.75 \\
1.54\end{array}$ & $\begin{array}{l}5.58 \\
1.56\end{array}$ & $\begin{array}{l}5.50 \\
1.45\end{array}$ & 17 & - & 67 & - & 17 & - & $\begin{array}{l}\text { Bite } \\
\text { Reptile }\end{array}$ & $\begin{array}{l}17 \\
17\end{array}$ \\
\hline & S & $\begin{array}{l}5.83 \\
1.47\end{array}$ & $\begin{array}{l}6.33 \\
0.78\end{array}$ & $\begin{array}{l}4.83 \\
1.70\end{array}$ & $\begin{array}{l}6.58 \\
0.67\end{array}$ & 75 & 081 & 17 & - & - & - & $\begin{array}{l}\text { Grass } \\
\text { Bite } \\
\text { Slimy }\end{array}$ & $\begin{array}{l}25 \\
17 \\
17\end{array}$ \\
\hline \multirow[t]{2}{*}{ Snap } & B & $\begin{array}{l}6.50 \\
1.00\end{array}$ & $\begin{array}{l}6.42 \\
0.67\end{array}$ & $\begin{array}{l}4.92 \\
1.31\end{array}$ & $\begin{array}{l}6.42 \\
0.79\end{array}$ & - & 100 & - & - & - & - & $\begin{array}{l}\text { Crackle } \\
\text { Finger } \\
\text { Pop }\end{array}$ & $\begin{array}{l}17 \\
17 \\
17\end{array}$ \\
\hline & $\mathrm{S}$ & $\begin{array}{l}5.67 \\
1.23\end{array}$ & $\begin{array}{l}4.83 \\
1.53\end{array}$ & $\begin{array}{l}4.42 \\
1.44\end{array}$ & $\begin{array}{l}5.25 \\
1.54\end{array}$ & 33 & $67-$ & - & - & - & - & $\begin{array}{l}\text { Crackle } \\
\text { Fingers }\end{array}$ & $\begin{array}{l}42 \\
25\end{array}$ \\
\hline \multirow[t]{2}{*}{ Sneeze } & B & $\begin{array}{l}6.75 \\
0.62\end{array}$ & $\begin{array}{l}6.08 \\
1.88\end{array}$ & $\begin{array}{l}4.83 \\
1.75\end{array}$ & $\begin{array}{l}6.17 \\
1.11\end{array}$ & - & 92 & 08 & - & - & - & $\begin{array}{l}\text { Cold } \\
\text { Allergy } \\
\text { Cough }\end{array}$ & $\begin{array}{l}50 \\
17 \\
17\end{array}$ \\
\hline & $\mathbf{S}$ & $\begin{array}{l}6.08 \\
1.44\end{array}$ & $\begin{array}{l}5.75 \\
1.06\end{array}$ & $\begin{array}{l}4.25 \\
1.54\end{array}$ & $\begin{array}{l}5.75 \\
1.06\end{array}$ & 08 & 83 & 08 & - & - & - & $\begin{array}{l}\text { Cold } \\
\text { Cough }\end{array}$ & $\begin{array}{l}33 \\
33\end{array}$ \\
\hline Song & B & $\begin{array}{l}6.83 \\
0.39\end{array}$ & $\begin{array}{l}6.83 \\
0.39\end{array}$ & $\begin{array}{l}6.17 \\
0.83\end{array}$ & $\begin{array}{l}6.75 \\
0.62\end{array}$ & - & 100 & - & - & - & - & $\begin{array}{l}\text { Music } \\
\text { Dance } \\
\text { Lyric } \\
\text { Sing }\end{array}$ & $\begin{array}{l}25 \\
17 \\
17 \\
17\end{array}$ \\
\hline
\end{tabular}


APPENDIX (Continued)

\begin{tabular}{|c|c|c|c|c|c|c|c|c|c|c|c|c|c|}
\hline \multirow[b]{2}{*}{ Noun } & \multirow{2}{*}{$\begin{array}{l}\text { Subject } \\
\text { Group }\end{array}$} & \multirow[b]{2}{*}{$\mathrm{f}$} & \multirow[b]{2}{*}{ c } & \multirow[b]{2}{*}{$\mathbf{m}$} & \multirow[b]{2}{*}{$\mathrm{i}$} & \multicolumn{6}{|c|}{ Modality (\%) } & \multirow{2}{*}{$\begin{array}{l}\text { Strongest } \\
\text { Associates }\end{array}$} & \multirow[b]{2}{*}{$\%$} \\
\hline & & & & & & $(\mathrm{v}$ & a & tc & ts & sp & sm) & & \\
\hline & s & $\begin{array}{l}6.17 \\
0.94\end{array}$ & $\begin{array}{l}5.58 \\
1.44\end{array}$ & $\begin{array}{l}6.00 \\
0.95\end{array}$ & $\begin{array}{l}6.08 \\
0.79\end{array}$ & 08 & 83 & - & - & 08 & - & $\begin{array}{l}\text { Sing } \\
\text { Dance }\end{array}$ & $\begin{array}{l}33 \\
25\end{array}$ \\
\hline \multirow[t]{2}{*}{ Soul } & B & $\begin{array}{l}6.83 \\
0.39\end{array}$ & $\begin{array}{l}2.42 \\
1.83\end{array}$ & $\begin{array}{l}3.83 \\
1.90\end{array}$ & $\begin{array}{l}1.92 \\
2.15\end{array}$ & - & - & - & - & 08 & - & $\begin{array}{l}\text { Spirit } \\
\text { Being }\end{array}$ & $\begin{array}{l}25 \\
17\end{array}$ \\
\hline & $\mathrm{s}$ & $\begin{array}{l}5.58 \\
1.16\end{array}$ & $\begin{array}{l}2.75 \\
1.82\end{array}$ & $\begin{array}{l}5.17 \\
1.64\end{array}$ & $\begin{array}{l}3.50 \\
2.15\end{array}$ & 17 & 08 & 08 & - & 42 & - & $\begin{array}{l}\text { Music } \\
\text { Spirit }\end{array}$ & $\begin{array}{l}17 \\
17\end{array}$ \\
\hline \multirow[t]{2}{*}{ Speaker } & B & $\begin{array}{l}6.75 \\
0.62\end{array}$ & $\begin{array}{l}6.50 \\
0.80\end{array}$ & $\begin{array}{l}5.17 \\
1.11\end{array}$ & $\begin{array}{l}6.00 \\
1.86\end{array}$ & - & 83 & 08 & - & - & - & $\begin{array}{l}\text { Stereo } \\
\text { Voice }\end{array}$ & $\begin{array}{l}17 \\
17\end{array}$ \\
\hline & $\mathrm{s}$ & $\begin{array}{l}5.67 \\
1.44\end{array}$ & $\begin{array}{l}5.92 \\
1.16\end{array}$ & $\begin{array}{l}4.83 \\
1.53\end{array}$ & $\begin{array}{l}5.50 \\
1.31\end{array}$ & 75 & 25 & - & - & - & - & $\begin{array}{l}\text { Amplifier } \\
\text { of the hous }\end{array}$ & $\begin{array}{l}17 \\
\text { se } 17\end{array}$ \\
\hline \multirow[t]{2}{*}{ Squeak } & B & $\begin{array}{l}6.75 \\
0.62\end{array}$ & $\begin{array}{l}6.42 \\
1.00\end{array}$ & $\begin{array}{l}4.50 \\
1.93\end{array}$ & $\begin{array}{l}6.50 \\
0.80\end{array}$ & - & 100 & - & - & - & - & $\begin{array}{l}\text { Noise } \\
\text { Sound }\end{array}$ & $\begin{array}{l}25 \\
17\end{array}$ \\
\hline & $\mathrm{s}$ & $\begin{array}{l}5.50 \\
1.57\end{array}$ & $\begin{array}{l}5.00 \\
1.71\end{array}$ & $\begin{array}{l}4.75 \\
1.14\end{array}$ & $\begin{array}{l}5.75 \\
1.14\end{array}$ & 08 & 92 & - & - & - & - & $\begin{array}{l}\text { Mouse } \\
\text { House }\end{array}$ & $\begin{array}{l}58 \\
17\end{array}$ \\
\hline \multirow[t]{2}{*}{ Stain } & B & $\begin{array}{l}6.00 \\
1.81\end{array}$ & $\begin{array}{l}3.83 \\
2.25\end{array}$ & $\begin{array}{l}3.75 \\
1.76\end{array}$ & $\begin{array}{l}3.08 \\
2.54\end{array}$ & 33 & 08 & - & - & 08 & - & $\begin{array}{l}\text { Color } \\
\text { Dark }\end{array}$ & $\begin{array}{l}17 \\
17\end{array}$ \\
\hline & $\mathbf{s}$ & $\begin{array}{l}5.92 \\
1.24\end{array}$ & $\begin{array}{l}5.50 \\
1.24\end{array}$ & $\begin{array}{l}4.17 \\
1.70\end{array}$ & $\begin{array}{l}5.33 \\
1.30\end{array}$ & 100 & - & - & - & - & - & Mess & 17 \\
\hline \multirow[t]{2}{*}{ Star } & B & $\begin{array}{l}6.50 \\
1.00\end{array}$ & $\begin{array}{l}5.17 \\
1.90\end{array}$ & $\begin{array}{l}4.67 \\
2.02\end{array}$ & $\begin{array}{l}4.33 \\
2.46\end{array}$ & 33 & - & 08 & - & 33 & - & $\begin{array}{l}\text { Sky } \\
\text { Heaven } \\
\text { Night }\end{array}$ & $\begin{array}{l}42 \\
17 \\
17\end{array}$ \\
\hline & s & $\begin{array}{l}5.92 \\
1.16\end{array}$ & $\begin{array}{l}5.67 \\
1.30\end{array}$ & $\begin{array}{l}5.00 \\
1.65\end{array}$ & $\begin{array}{l}6.17 \\
0.58\end{array}$ & 100 & $0-$ & - & - & - & - & $\begin{array}{l}\text { Light } \\
\text { Moon } \\
\text { Sky }\end{array}$ & $\begin{array}{l}33 \\
17 \\
17\end{array}$ \\
\hline \multirow[t]{2}{*}{ string } & B & $\begin{array}{l}6.50 \\
0.90\end{array}$ & $\begin{array}{l}5.58 \\
1.68\end{array}$ & $\begin{array}{l}4.67 \\
2.02\end{array}$ & $\begin{array}{l}6.33 \\
0.98\end{array}$ & 17 & - & 75 & - & 08 & - & $\begin{array}{l}\text { Tie } \\
\text { Cord }\end{array}$ & $\begin{array}{l}33 \\
17\end{array}$ \\
\hline & $s$ & $\begin{array}{l}5.67 \\
1.15\end{array}$ & $\begin{array}{l}5.92 \\
1.31\end{array}$ & $\begin{array}{l}4.58 \\
1.31\end{array}$ & $\begin{array}{l}5.33 \\
1.30\end{array}$ & 83 & - & 08 & - & 08 & - & Rope & 17 \\
\hline \multirow[t]{2}{*}{ Sunset } & B & $\begin{array}{l}6.00 \\
1.71\end{array}$ & $\begin{array}{l}4.83 \\
2.41\end{array}$ & $\begin{array}{l}4.00 \\
2.00\end{array}$ & $\begin{array}{l}4.00 \\
2.45\end{array}$ & 25 & - & 17 & - & 25 & - & Evening & 25 \\
\hline & s & $\begin{array}{l}6.00 \\
0.95\end{array}$ & $\begin{array}{l}5.50 \\
1.24\end{array}$ & $\begin{array}{l}5.42 \\
1.16\end{array}$ & $\begin{array}{l}6.17 \\
0.83\end{array}$ & 100 & $0-$ & - & - & - & - & $\begin{array}{l}\text { Night } \\
\text { Sunrise }\end{array}$ & $\begin{array}{l}33 \\
17\end{array}$ \\
\hline
\end{tabular}


APPENDIX (Continued)

\begin{tabular}{|c|c|c|c|c|c|c|c|c|c|c|c|c|c|}
\hline \multirow[b]{2}{*}{ Noun } & \multirow{2}{*}{$\begin{array}{l}\text { Subject } \\
\text { Group }\end{array}$} & \multirow[b]{2}{*}{$\mathrm{f}$} & \multirow[b]{2}{*}{$\mathrm{c}$} & \multirow[b]{2}{*}{$\mathrm{m}$} & \multirow[b]{2}{*}{$\mathrm{i}$} & \multicolumn{6}{|c|}{ Modality (\%) } & \multirow{2}{*}{$\begin{array}{c}\text { Strongest } \\
\text { Associates }\end{array}$} & \multirow[b]{2}{*}{$\%$} \\
\hline & & & & & & $(\mathrm{v}$ & $\mathbf{a}$ & tc & ts & $\mathrm{sp}$ & $\mathrm{sm})$ & & \\
\hline \multirow[t]{2}{*}{ Teacher } & B & $\begin{array}{l}6.83 \\
0.39\end{array}$ & $\begin{array}{l}5.92 \\
1.56\end{array}$ & $\begin{array}{l}5.75 \\
1.06\end{array}$ & $\begin{array}{l}5.17 \\
2.44\end{array}$ & 08 & $67-$ & - & - & 08 & - & $\begin{array}{l}\text { Student } \\
\text { Instructor } \\
\text { Professor } \\
\text { Pupil }\end{array}$ & $\begin{array}{l}25 \\
17 \\
17 \\
17\end{array}$ \\
\hline & $\mathrm{s}$ & $\begin{array}{l}6.25 \\
0.75\end{array}$ & $\begin{array}{l}5.75 \\
1.22\end{array}$ & $\begin{array}{l}5.67 \\
1.07\end{array}$ & $\begin{array}{l}5.33 \\
1.44\end{array}$ & 75 & $25-$ & - & - & - & - & $\begin{array}{l}\text { Professor } \\
\text { Pet }\end{array}$ & $\begin{array}{l}25 \\
17\end{array}$ \\
\hline \multirow[t]{2}{*}{ Tendency } & B & $\begin{array}{l}6.17 \\
0.72\end{array}$ & $\begin{array}{l}2.25 \\
1.66\end{array}$ & $\begin{array}{l}3.08 \\
1.62\end{array}$ & $\begin{array}{l}2.17 \\
2.17\end{array}$ & - & 08 & - & - & 08 & - & $\begin{array}{l}\text { Inclined } \\
\text { Prone }\end{array}$ & $\begin{array}{l}17 \\
17\end{array}$ \\
\hline & $\mathrm{S}$ & $\begin{array}{l}5.17 \\
1.34\end{array}$ & $\begin{array}{l}1.83 \\
0.58\end{array}$ & $\begin{array}{l}4.17 \\
1.40\end{array}$ & $\begin{array}{l}1.33 \\
0.89\end{array}$ & 08 & - & - & - & 08 & - & Suicidal & 17 \\
\hline \multirow[t]{2}{*}{ Thunder } & B & $\begin{array}{l}6.83 \\
0.39\end{array}$ & $\begin{array}{l}6.58 \\
0.67\end{array}$ & $\begin{array}{l}5.25 \\
1.60\end{array}$ & $\begin{array}{l}6.67 \\
0.49\end{array}$ & - & 100 & - & - & - & - & $\begin{array}{l}\text { Lightning } \\
\text { Loud }\end{array}$ & $\begin{array}{l}58 \\
17\end{array}$ \\
\hline & $\mathrm{s}$ & $\begin{array}{l}6.00 \\
0.95\end{array}$ & $\begin{array}{l}5.92 \\
1.24\end{array}$ & $\begin{array}{l}5.00 \\
1.54\end{array}$ & $\begin{array}{l}6.00 \\
0.85\end{array}$ & - & 920 & 08 & - & - & - & Lightning & 83 \\
\hline \multirow[t]{2}{*}{ Tone } & B & $\begin{array}{l}6.58 \\
0.80\end{array}$ & $\begin{array}{l}6.08 \\
1.31\end{array}$ & $\begin{array}{l}5.58 \\
1.51\end{array}$ & $\begin{array}{l}5.75 \\
1.91\end{array}$ & - & 100 & - & - & - & - & $\begin{array}{l}\text { Sound } \\
\text { Music }\end{array}$ & $\begin{array}{l}25 \\
17\end{array}$ \\
\hline & $\mathrm{S}$ & $\begin{array}{l}5.58 \\
1.24\end{array}$ & $\begin{array}{l}4.00 \\
1.81\end{array}$ & $\begin{array}{l}4.17 \\
1.95\end{array}$ & $\begin{array}{l}5.25 \\
1.42\end{array}$ & - & 100 & - & - & - & - & $\begin{array}{l}\text { Pitch } \\
\text { Muscles } \\
\text { Music }\end{array}$ & $\begin{array}{l}17 \\
17 \\
17\end{array}$ \\
\hline \multirow[t]{2}{*}{ Tower } & B & $\begin{array}{l}6.50 \\
0.80\end{array}$ & $\begin{array}{l}5.58 \\
2.11\end{array}$ & $\begin{array}{l}4.42 \\
1.78\end{array}$ & $\begin{array}{l}5.08 \\
2.23\end{array}$ & 17 & -1 & 17 & - & 50 & - & $\begin{array}{l}\text { High } \\
\text { Tall }\end{array}$ & $\begin{array}{l}50 \\
33\end{array}$ \\
\hline & s & $\begin{array}{l}5.58 \\
1.16\end{array}$ & $\begin{array}{l}5.67 \\
1.07\end{array}$ & $\begin{array}{l}5.00 \\
1.13\end{array}$ & $\begin{array}{l}5.50 \\
1.45\end{array}$ & 100 & - & - & - & - & - & $\begin{array}{l}\text { Building } \\
\text { Pisa }\end{array}$ & $\begin{array}{l}25 \\
17\end{array}$ \\
\hline \multirow[t]{2}{*}{ Troops } & B & $\begin{array}{l}6.25 \\
0.97\end{array}$ & $\begin{array}{l}4.92 \\
1.98\end{array}$ & $\begin{array}{l}4.67 \\
1.50\end{array}$ & $\begin{array}{l}4.83 \\
2.17\end{array}$ & 08 & 50 & - & - & 25 & - & $\begin{array}{l}\text { Army } \\
\text { March } \\
\text { Military }\end{array}$ & $\begin{array}{l}58 \\
17 \\
17\end{array}$ \\
\hline & S & $\begin{array}{l}5.00 \\
1.28\end{array}$ & $\begin{array}{l}5.33 \\
1.30\end{array}$ & $\begin{array}{l}4.67 \\
1.56\end{array}$ & $\begin{array}{l}5.08 \\
1.78\end{array}$ & 75 & 17 & - & - & - & - & $\begin{array}{l}\text { Army } \\
\text { War }\end{array}$ & $\begin{array}{l}50 \\
25\end{array}$ \\
\hline \multirow[t]{2}{*}{ Trumpet } & $\mathrm{B}$ & $\begin{array}{l}6.17 \\
1.19\end{array}$ & $\begin{array}{l}6.33 \\
0.89\end{array}$ & $\begin{array}{l}5.33 \\
1.50\end{array}$ & $\begin{array}{l}6.33 \\
0.98\end{array}$ & - & 100 & - & - & - & - & $\begin{array}{l}\text { Instrument } \\
\text { Blow } \\
\text { Sound }\end{array}$ & $\begin{array}{l}42 \\
17 \\
17\end{array}$ \\
\hline & $\mathrm{S}$ & $\begin{array}{l}5.33 \\
1.67\end{array}$ & $\begin{array}{l}5.92 \\
0.90\end{array}$ & $\begin{array}{l}5.17 \\
1.19\end{array}$ & $\begin{array}{l}5.58 \\
1.44\end{array}$ & 50 & 50 & - & - & - & - & $\begin{array}{l}\text { Instrument } \\
\text { Player }\end{array}$ & $\begin{array}{l}17 \\
17\end{array}$ \\
\hline Tweezers & B & $\begin{array}{l}6.17 \\
0.83\end{array}$ & $\begin{array}{l}5.75 \\
1.42\end{array}$ & $\begin{array}{l}5.17 \\
1.19\end{array}$ & $\begin{array}{l}6.08 \\
1.24\end{array}$ & 08 & - & 92 & - & - & - & Eyebrows & 33 \\
\hline
\end{tabular}


APPENDIX (Continued)

\begin{tabular}{|c|c|c|c|c|c|c|c|c|c|c|c|c|c|}
\hline \multirow[b]{2}{*}{ Noun } & \multirow{2}{*}{$\begin{array}{l}\text { Subject } \\
\text { Group }\end{array}$} & \multirow[b]{2}{*}{$\mathrm{f}$} & \multirow[b]{2}{*}{ c } & \multirow[b]{2}{*}{$\mathrm{m}$} & \multirow[b]{2}{*}{$\mathrm{i}$} & \multicolumn{6}{|c|}{ Modality (\%) } & \multirow{2}{*}{$\begin{array}{c}\text { Strongest } \\
\text { Associates }\end{array}$} & \multirow[b]{2}{*}{$\%$} \\
\hline & & & & & & $(\mathrm{v}$ & $\mathrm{a}$ & tc & ts & $\mathrm{sp}$ & $\mathrm{sm})$ & & \\
\hline & $s$ & $\begin{array}{l}5.08 \\
1.68\end{array}$ & $\begin{array}{l}6.25 \\
0.87\end{array}$ & $\begin{array}{l}4.00 \\
1.60\end{array}$ & $\begin{array}{l}5.50 \\
1.38\end{array}$ & 83 & - & 17 & - & - & - & Pluck & 42 \\
\hline \multirow[t]{2}{*}{ Valley } & B & $\begin{array}{l}6.25 \\
1.22\end{array}$ & $\begin{array}{l}4.83 \\
2.17\end{array}$ & $\begin{array}{l}5.00 \\
1.65\end{array}$ & $\begin{array}{l}5.33 \\
1.72\end{array}$ & 25 & - & 08 & - & 58 & - & $\begin{array}{l}\text { Mountain } \\
\text { Hill } \\
\text { Low }\end{array}$ & $\begin{array}{l}33 \\
17 \\
17\end{array}$ \\
\hline & $\mathrm{S}$ & $\begin{array}{l}5.17 \\
1.75\end{array}$ & $\begin{array}{l}5.33 \\
1.37\end{array}$ & $\begin{array}{l}4.75 \\
1.42\end{array}$ & $\begin{array}{l}5.58 \\
1.24\end{array}$ & 92 & - & - & - & 08 & - & $\begin{array}{l}\text { Hills } \\
\text { Death } \\
\text { Mountain }\end{array}$ & $\begin{array}{l}25 \\
17 \\
17\end{array}$ \\
\hline \multirow[t]{2}{*}{ Vapor } & B & $\begin{array}{l}6.50 \\
0.67\end{array}$ & $\begin{array}{l}4.92 \\
2.02\end{array}$ & $\begin{array}{l}4.42 \\
1.73\end{array}$ & $\begin{array}{l}5.17 \\
1.85\end{array}$ & 08 & - & 75 & - & - & - & $\begin{array}{l}\text { Water } \\
\text { Gas }\end{array}$ & $\begin{array}{l}33 \\
17\end{array}$ \\
\hline & $\mathrm{s}$ & $\begin{array}{l}5.42 \\
1.24\end{array}$ & $\begin{array}{l}4.50 \\
1.62\end{array}$ & $\begin{array}{l}3.92 \\
1.78\end{array}$ & $\begin{array}{l}4.58 \\
1.73\end{array}$ & 42 & - & 08 & - & 17 & 25 & $\begin{array}{l}\text { Gas } \\
\text { Action } \\
\text { Mist }\end{array}$ & $\begin{array}{l}25 \\
17 \\
17\end{array}$ \\
\hline \multirow[t]{2}{*}{ Virtue } & B & $\begin{array}{l}6.25 \\
0.75\end{array}$ & $\begin{array}{l}2.08 \\
1.68\end{array}$ & $\begin{array}{l}3.25 \\
1.76\end{array}$ & $\begin{array}{l}1.92 \\
2.15\end{array}$ & - & - & - & - & 08 & - & $\begin{array}{l}\text { Honesty } \\
\text { Quality }\end{array}$ & $\begin{array}{l}17 \\
17\end{array}$ \\
\hline & $\mathrm{S}$ & $\begin{array}{l}5.17 \\
1.47\end{array}$ & $\begin{array}{l}2.00 \\
0.95\end{array}$ & $\begin{array}{l}3.75 \\
1.66\end{array}$ & $\begin{array}{l}2.00 \\
1.91\end{array}$ & 08 & - & - & - & 25 & - & Honor & 17 \\
\hline \multirow[t]{2}{*}{ Whisper } & B & $\begin{array}{l}6.67 \\
0.65\end{array}$ & $\begin{array}{l}6.08 \\
1.24\end{array}$ & $\begin{array}{l}5.25 \\
2.63\end{array}$ & $\begin{array}{l}6.25 \\
1.06\end{array}$ & - & 100 & - & - & - & - & $\begin{array}{l}\text { Soft } \\
\text { Quiet } \\
\text { Secret }\end{array}$ & $\begin{array}{l}42 \\
17 \\
17\end{array}$ \\
\hline & S & $\begin{array}{l}5.92 \\
1.16\end{array}$ & $\begin{array}{l}5.08 \\
1.51\end{array}$ & $\begin{array}{l}4.92 \\
1.16\end{array}$ & $\begin{array}{l}5.42 \\
1.31\end{array}$ & - & 100 & - & - & - & - & Quiet & 33 \\
\hline \multirow[t]{2}{*}{ Whistle } & B & $\begin{array}{l}6.75 \\
0.45\end{array}$ & $\begin{array}{l}6.33 \\
0.78\end{array}$ & $\begin{array}{l}5.42 \\
1.00\end{array}$ & $\begin{array}{l}6.58 \\
0.90\end{array}$ & - & 100 & - & - & - & - & $\begin{array}{l}\text { Blow } \\
\text { Police }\end{array}$ & $\begin{array}{l}25 \\
17\end{array}$ \\
\hline & $\mathrm{S}$ & $\begin{array}{l}5.42 \\
1.62\end{array}$ & $\begin{array}{l}5.92 \\
1.08\end{array}$ & $\begin{array}{l}4.67 \\
1.07\end{array}$ & $\begin{array}{l}5.50 \\
1.00\end{array}$ & 08 & 92 & - & - & - & - & $\begin{array}{l}\text { Blow } \\
\text { Work }\end{array}$ & $\begin{array}{l}25 \\
25\end{array}$ \\
\hline \multirow[t]{2}{*}{ Wigwam } & B & $\begin{array}{l}6.08 \\
1.16\end{array}$ & $\begin{array}{l}4.83 \\
2.04\end{array}$ & $\begin{array}{l}3.25 \\
2.26\end{array}$ & $\begin{array}{l}5.17 \\
1.70\end{array}$ & 17 & 08 & 33 & - & 33 & - & Indian & 92 \\
\hline & S & $\begin{array}{l}4.33 \\
1.44\end{array}$ & $\begin{array}{l}5.83 \\
1.11\end{array}$ & $\begin{array}{l}4.58 \\
1.44\end{array}$ & $\begin{array}{l}5.83 \\
1.19\end{array}$ & 100 & - & - & - & - & - & $\begin{array}{l}\text { Indian } \\
\text { Teepee }\end{array}$ & $\begin{array}{l}50 \\
17\end{array}$ \\
\hline
\end{tabular}

